\title{
SPHERE view of the jet and the envelope of RY Tauri ${ }^{\star}$
}

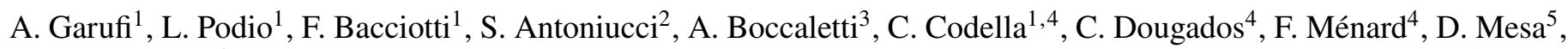 \\ M. Meyer ${ }^{6}$, B. Nisini ${ }^{2}$, H. M. Schmid ${ }^{7}$, T. Stolker ${ }^{7}$, J. L. Baudino ${ }^{8}$, B. Biller ${ }^{9}$, M. Bonavita ${ }^{9}$, M. Bonnefoy ${ }^{4}$, \\ F. Cantalloube ${ }^{10}$, G. Chauvin ${ }^{4}$, A. Cheetham ${ }^{11}$, S. Desidera ${ }^{5}$, V. D'Orazi ${ }^{5}$, M. Feldt ${ }^{10}$, R. Galicher ${ }^{3}$, A. Grandjean ${ }^{4}$, \\ R. Gratton ${ }^{5}$, J. Hagelberg ${ }^{7}$, A. M. Lagrange ${ }^{4}$, M. Langlois ${ }^{12}$, J. Lannier ${ }^{4}$, C. Lazzoni ${ }^{13}$, A. L. Maire ${ }^{14}$, C. Perrot ${ }^{3,15,16}$, \\ E. Rickman ${ }^{11}$, T. Schmidt ${ }^{3}$, A. Vigan ${ }^{17}$, A. Zurlo ${ }^{17,18,19}$, A. Delboulbé ${ }^{4}$, D. Le Mignant ${ }^{17}$, D. Fantinel ${ }^{5}$, \\ O. Möller-Nilsson ${ }^{10}$, L. Weber ${ }^{11}$, and J.-F. Sauvage ${ }^{17}$ \\ (Affiliations can be found after the references)
}

Received 26 March 2019 / Accepted 17 June 2019

\begin{abstract}
Context. Jets are rarely associated with pre-main sequence intermediate-mass stars. This contrasts with the frequent detection of jets in lower mass or younger stars. Optical and near-IR observations of jet-driving sources are often hindered by the presence of a natal envelope.

Aims. Jets around partly embedded sources are a useful diagnostic to constrain the geometry of the concealed protoplanetary disk. We intend to clarify how the jet-driving mechanisms are affected by both spatial anisotropies and episodic variations at the (sub-)au scale from the star.

Methods. We obtained a rich set of high-contrast VLT/SPHERE observations from 0.6 to $2.2 \mu \mathrm{m}$ of the young intermediate-mass star RY Tau. Given the proximity to the Sun of this source, our images have the highest spatial resolution ever obtained for an atomic jet (down to $\sim 4$ au).

Results. Optical observations in polarized light show no sign of the protoplanetary disk detected by ALMA. Instead, we observed a diffuse signal resembling a remnant envelope with an outflow cavity. The jet is detected in the $\mathrm{H} \alpha$, [S II] at $1.03 \mu \mathrm{m}, \mathrm{He}$ I at $1.08 \mu \mathrm{m}$, and [Fe II] lines in the $1.25 \mu \mathrm{m}$ and $1.64 \mu \mathrm{m}$. The jet appears to be wiggling and its radial width increasing with the distance is complementary to the shape of the outflow cavity suggesting a strong interaction with jet and envelope. Through the estimated tangential velocity $\left(\sim 100 \mathrm{~km} \mathrm{~s}^{-1}\right)$, we revealed a possible connection between the launching time of the jet substructures and the stellar activity of RY Tau.

Conclusions. RY Tau is at an intermediate stage toward the dispersal of the natal envelope. This source shows episodic increases of mass accretion and ejection similarly to other known intermediate-mass stars. The amount of observed jet wiggle is consistent with the presence of a precessing disk warp or misaligned inner disk that would be induced by an unseen planetary or sub-stellar companion at sub- or few-au scales respectively. The high disk mass of RY Tau and of two other jet-driving intermediate-mass stars, HD 163296 and MWC480, suggests that massive, full disks are more efficient at launching prominent jets.
\end{abstract}

Key words. ISM: jets and outflows - planet-disk interactions - stars: variables: T Tauri, Herbig Ae/Be - protoplanetary disks infrared: stars

\section{Introduction}

The formation history of planets is closely connected to the global evolution of protoplanetary disks. Our knowledge of disk morphology has greatly advanced in the last few years thanks to the high-contrast and high-resolution imaging in both the visible and near-IR (NIR) and the (sub-)mm offered by new instruments like Spectro-Polarimetric High-contrast Exoplanet REsearch instrument at the Very Large Telescope (VLT/SPHERE), Gemini Planet Imager (GPI), and Atacama Large Millimeter Array (ALMA). However, if on one hand ALMA also has access to younger, embedded sources (see, e.g., Sheehan \& Eisner 2017), the optical and NIR imaging has important limitations in this regard because of the much higher extinction of these young sources. In fact, the protoplanetary disks of well-studied premain sequence (PMS) stars like HL Tau (Takami et al. 2007)

\footnotetext{
${ }^{\star}$ Based on observations performed with VLT/SPHERE under program ID 096.C-0241(C), 096.C-0241(F), 096.C-0248(A), 096.C0454(A), and 1100.C-0481(A).
}

or DG Tau B (Podio et al. 2011) have not been imaged at short wavelengths, and the whole sample of disks with available NIR images is biased toward more evolved sources with no remnant envelope (Garufi et al. 2018).

Among the various physical processes that affect the morphology of protoplanetary disks, the removal of mass and angular momentum through jets and outflows may play a fundamental role (see the review by Frank et al. 2014) since it can limit the increase of stellar rotation and allows a high accretion rate for a large fraction of the protoplanetary disk lifetime. Given the importance of this element for the star formation process, it is crucial to establish if the ejection of jets is a general phenomenon for forming stars of all masses and evolutionary stages. Jets are also a useful diagnostic of the innermost disk regions being closely connected to the accretion processes and the interplay of the star and disk.

It is known that jets are almost ubiquitous in protostars (see, e.g., Nisini et al. 2015). However, jets are often observed in more evolved PMS stars, and in particular around the low-mass 
( $\lesssim 1.5 M_{\odot}$ ) T Tau stars (TTSs, e.g., Hartigan et al. 1995; Bacciotti et al. 2002). The observations of these stars are facilitated by their intrinsic abundance and by their long PMS timescale that allow the direct imaging of their disk after their parent envelope is dissipated. These low-mass stars preserve their outer convective zones until and beyond the zero-age main sequence (ZAMS), and are thus accompanied by strong magnetic field. On the other hand, intermediate-mass stars $\left(1.5-5 M_{\odot}\right)$ develop a radiative envelope throughout their PMS stage (Gregory et al. 2012). Observationally, these stars have an initially late spectral type $(K-F)$ and are referred to as intermediate-mass TTSs (IMTTSs, see e.g., Calvet et al. 2004), while at a later stage of their PMS evolution they appear as Herbig AeBe stars and eventually turn into A/B main sequence stars. The magnetic field of Herbig stars is weaker and less characterized (Hubrig et al. 2018). Unlike TTSs, observations of jets around intermediateand high-mass young stars are rare, although a few examples exist (e.g., Devine et al. 2000; Antoniucci et al. 2016).

RY Tau is an excellent laboratory for the study of jets around intermediate-mass stars and of the later stages of envelope dispersal. This source is in fact associated to an extended nebulosity and a remnant envelope close to the star (Nakajima \& Golimowski 1995; Takami et al. 2013) as well as to an optically bright jet (Cabrit et al. 1990; St-Onge \& Bastien 2008; Agra-Amboage et al. 2009; Coffey et al. 2015; Skinner et al. 2018). High-amplitude photospheric variations (Petrov et al. 1999) and the primordial spectral energy distribution (SED, Robitaille et al. 2007) also point to a relatively young source. However, its protoplanetary disk shows rings and an inner cavity in the sub-millimeter (Isella et al. 2010; Long et al. 2018) that are typically observed in more evolved sources.

In this work, we present new optical and NIR observations of RY Tau obtained with VLT/SPHERE (Beuzit et al. 2019). Although this instrument was designed and optimized for planet and disk characterization, it also allows us to image the inner regions of jets in the atomic lines at an unprecedented resolution of $\sim 30$ mas, corresponding to $4-5$ au at $130 \mathrm{pc}$. The paper is organized as follows. In Sect. 2, we describe the analyzed datasets. Then, in Sects. 3 and 4 we present our results from spectroscopic, photometric, and imaging data. Finally, in Sects. 5 and 6 , we discuss our findings and conclude.

\section{Observations and data reduction}

\subsection{VLT/SPHERE images}

Multiple images of RY Tau were taken in the context of the guaranteed time observations of the high-contrast imager SPHERE (Beuzit et al. 2019), operating at the VLT in conjunction with the SPHERE AO for eXoplanet Observation (SAXO) system (Fusco et al. 2006). The following techniques were employed.

Differential polarization imaging (DPI). Polarimetric observations of RY Tau were taken with the sub-instrument ZIMPOL (Zurich imaging polarimeter, Schmid et al. 2018) in the $I^{\prime}$ band $\left(\lambda_{\mathrm{c}}=790 \mathrm{~nm}\right)$ on 2015 December 17. To minimize instrument artifacts, the observations were performed in DPI field stabilized mode by alternating the object orientation by $60^{\circ}$ and dithering its position by 14 pixels. We operated in both SlowPolarimetry mode, which maximizes the signal-to-noise of the outer regions, and FastPolarimetry mode, which provides the highest polarimetric precision of the inner regions. In Slow Polarimetry mode we adopted a 155 mas-diameter Lyot coronagraph and DIT $=50 \mathrm{~s}$, while in FastPolarimetry a DIT $=20 \mathrm{~s}$ and no coronagraph were employed. The DPI data reduction follows the standard technique adopted in several works involving NAOS-CONICA (NACO) and SPHERE observations (see, e.g., Avenhaus et al. 2014; Garufi et al. 2016) and provides as a final product the Stokes parameters $Q$ and $U$, as well as the polarized intensity $P I=\left(Q^{2}+U^{2}\right)^{1 / 2}$ and the radial parameters $Q_{\phi}$ and $U_{\phi}$ (Schmid et al. 2006).

Reference-star differential imaging (RDI). ZIMPOL observations of RY Tau in the $I^{\prime}$ band were also taken in Classical Imaging (CI) on 2015 December 17, along with those of a reference star, HD 284071. Observations were taken in pupil stabilized mode with DIT $=10 \mathrm{sec}$. The reduction followed the basic steps of the ZIMPOL/DPI reduction, leading to the extraction of 70 individual intensity frames for both RY Tau and the reference star that were scaled, subtracted, and averaged to generate a unique residual image.

Spectral differential imaging (SDI). RY Tau was also observed with ZIMPOL on 2015 November 8 in the $\mathrm{H} \alpha\left(\lambda_{\mathrm{c}}=\right.$ $655.6 \mathrm{~nm}, \Delta \lambda=5.5 \mathrm{~nm})$ and in the adjacent continuum $\left(\lambda_{\mathrm{c}}=\right.$ $644.9 \mathrm{~nm}, \Delta \lambda=4.1 \mathrm{~nm}$ ) filters (Program ID 096.C-0454(A), PI: Dougados). Images were acquired in field-stabilized mode with no coronagraph for a total integration time on source of $47 \mathrm{~min}$ utes. The data were processed using the ETH Zurich ZIMPOL IDL pipeline (version 1.5), which applies standard bias, dark, and flat-field corrections, and allows for re-centering, derotation, and combination of the frames. The data reduction followed the same procedure described by Antoniucci et al. (2016). In particular, we first normalized each continuum frame to the corresponding $\mathrm{H} \alpha$ frame to take into account the different filter band-passes, using the ratio of the total counts measured in the star peak region in the two images as normalization factor. Then, we performed a frame-by-frame subtraction of the $\mathrm{H} \alpha$ and continuum exposures to remove the continuum emission. All the continuum-subtracted frames thus obtained were eventually de-rotated and averaged to generate the final $\mathrm{H} \alpha$ image.

Integral field spectroscopy (IFS) and dual band imaging (DBI). Finally, RY Tau was observed as part of the SpHere INfrared survey for Exoplanets (SHINE, Chauvin et al. 2017) four times between 2015 October 27 and 2017 November 29. Observations consist of simultaneous imaging with the infrared dual-band imager and spectrograph (IRDIS, Dohlen et al. 2008) and integral field spectroscopy instrument (IFS, Claudi et al. 2008). They were done in both IRDIFS and IRDIFS_EXT modes, that is with IFS operating between $Y$ and $J$ spectral bands $(\lambda=0.95-1.35 \mu \mathrm{m})$ and IRDIS simultaneously performing DBI (Vigan et al. 2010) in $H 23$ band $\left(\lambda_{\mathrm{c}}=1.58 / 1.67 \mu \mathrm{m}\right.$, $\Delta \lambda=52 / 54 \mathrm{~nm})$ and with IFS operating between $Y$ and $H$ bands $(\lambda=0.95-1.65 \mu \mathrm{m})$ and IRDIS in DBI in $K 12$ band $\left(\lambda_{\mathrm{c}}=\right.$ $2.10 / 2.25 \mu \mathrm{m}, \Delta \lambda=102 / 109 \mathrm{~nm})$, respectively. The spectral resolution is $\sim 50$ in the IRDIFS and $\sim 30$ in the IRDIFS_EXT modes. All observations were pupil stabilized to enable angular differential imaging (ADI) and the star was centered under an apodized pupil Lyot coronagraph (Carbillet et al. 2011) of radius $r=185$ mas. All IFS and IRDIS datasets were reduced with the SPHERE Data Reduction and Handling (DRH) pipeline (Pavlov et al. 2008) in the SPHERE Data Center (DC ${ }^{1}$, Delorme et al. 2017). The fully reduced IFS images were combined to four-dimensional data cubes (two spatial, one spectral, and one time dimension) and further processed with the SHINE Specal pipeline (Galicher et al. 2018). The latter performs angular and spectral differential imaging (ADI+SDI) in the form of classical ADI (cADI, Marois et al. 2006), template locally optimized combination of images (TLOCI, Marois et al. 2014), and

http://sphere.osug.fr/spip.php?rubrique16\&lang=en 
Table 1. Summary of observations.

\begin{tabular}{|c|c|c|c|c|c|c|c|}
\hline Instrument & Mode & Waveband & Date & $t_{\exp }(\min )$ & $\theta$ (mas) & Main target emission & Figures \\
\hline \multirow{4}{*}{ IRDIS } & \multirow{4}{*}{ DBI } & $H 23$ & $2015-10-27$ & 68 & 60 & Continuum, [Fe II] line & 2 (top panels), 3,4 \\
\hline & & $K 12$ & $2015-12-26$ & 51 & 80 & Continuum, $\mathrm{H}_{2}$ line & - \\
\hline & & $K 12$ & 2016-01-01 & 85 & 80 & Continuum, $\mathrm{H}_{2}$ line & - \\
\hline & & $H 23$ & $2017-11-29$ & 102 & 60 & Continuum, [Fe II] line & 2 (top panels), 3 \\
\hline \multirow{4}{*}{ IFS } & \multirow{4}{*}{ IFS } & $Y J$ & $2015-10-27$ & 68 & 40 & [S II], He I, [Fe II] lines & - \\
\hline & & $Y H$ & $2015-12-26$ & 51 & 50 & [S II $],$ He I, [Fe II] lines & - \\
\hline & & $Y H$ & 2016-01-01 & 85 & 50 & [S II], He I, [Fe II] lines & - \\
\hline & & $Y J$ & $2017-11-29$ & 102 & 40 & [S II], He I, [Fe II] lines & 2 (bottom left panels) \\
\hline \multirow{3}{*}{ ZIMPOL } & DPI & $I^{\prime}$ & $2015-12-17$ & $40+16$ & 30 & Polarized continuum & 1,4 \\
\hline & RDI & $I^{\prime}$ & $2015-12-27$ & $11+11$ & 30 & Continuum & 1 \\
\hline & SDI & $\mathrm{H} \alpha$ & 2015-11-08 & 47 & 30 & $\mathrm{H} \alpha$ & 2 (bottom right panel), \\
\hline
\end{tabular}

Notes. Columns are: instrument, observing mode, date, waveband, exposure time, angular resolution, main target emission, and relative figure in this work. DBI: dual band imaging, IFS: integral field spectroscopy, DPI: differential polarization imaging, RDI: reference-star differential imaging, SDI: spectral differential imaging.

Table 2. Stellar properties from this work.

\begin{tabular}{ll}
\hline \hline Distance & $133 \mathrm{pc}$ \\
Effective temperature & $5750 \mathrm{~K}$ \\
Extinction $A_{\mathrm{V}}$ & $1.5 \pm 0.7 \mathrm{mag}$ \\
Luminosity & $6.3_{-3.2}^{+9.1} L_{\odot}$ \\
Mass & $\sim 1.9 M_{\odot}$ \\
Age & $\sim 4.5 \mathrm{Myr}$ \\
\hline
\end{tabular}

principal component analysis (PCA, Soummer et al. 2012; Amara et al. 2015). SDI is performed on the IFS images by constructing a median PSF from all available channels and by removing this reference image from each individual channel, so as to enhance the contrast of line emission compared to continuum emission.

All SPHERE images have been corrected for the SPHERE true north of $-1.8^{\circ}$ (Maire et al. 2016). The angular resolution achieved is approximately 30 mas for the ZIMPOL images, 40-50 mas for the IFS images, and 60-80 mas for the IRDIS images. A summary of observations is shown in Table 1.

\subsection{WHT/UES spectra}

Constraints on the stellar effective temperature can be obtained from optical high-resolution spectra. In this work, we made use of four spectra of RY Tau that were obtained on 1998 Oct 25 and 1999 Jan 28, 29, and 31 with the 4.2-m William Herschel Telescope (WHT) on the Observatorio del Roque de los Muchachos (La Palma, Spain) equipped with the Utrecht Echelle Spectrograph (UES). The observations belong to the International Time program Export (Eiroa et al. 2000). UES was set to provide a wavelength coverage between 3800 and $5900 \AA$. The spectra were dispersed into 59 echelle orders with a resolving power of 49000 . The slit width was set to $1.15^{\prime \prime}$ projected on the sky. Details about the reduction of the spectra can be found in Mora et al. (2001).

\section{Stellar properties}

The stellar properties of RY Tau from the literature are often discrepant. In this section, we perform their new calculation and discuss the critical estimate of the distance to the source. These parameters are summarized in Table 2.

\subsection{Distance}

RY Tau belongs to the Taurus star-forming region (Kenyon et al. 1994) and it has been typically assumed to be at $133_{-30}^{+55} \mathrm{pc}$, based on the HIPPARCOS data (ESA 1997). However, TychoGaia Astrometric Solution (TGAS) from Gaia DR1 (Gaia Collaboration 2016) revealed $d=176_{-23}^{+32} \mathrm{pc}$ and Gaia DR2 (Gaia Collaboration 2018) $d=442_{-42}^{+53}$ pc. The large inconsistency between the HIPPARCOS and Gaia estimates raised some doubts about the latter. DR2 is in fact still a preliminary release, where several data processing loops are not closed and various systematic effects are still present (Lindegren et al. 2018).

To test the possibility that the Gaia measurement is erroneous, we looked at 29 other members of its (visual) parent cloud, L1495 (see Luhman 2018). All these sources have $d$ between 119.5 and $139.1 \mathrm{pc}(\bar{d}=129.2 \mathrm{pc})$ and an average proper motion of 8.97 mas $(\sigma=1.27)$ and $-24.97(\sigma=1.26)$ mas in right ascension and declination. RY Tau shows a consistent proper motion of 9.08 and -23.05 from HIPPARCOS and of 9.10 and -25.86 from Gaia DR1, while the DR2 shows a slightly discrepant value $(4.98,-25.71)$.

Given these premises, we are inclined to consider the Gaia DR2 measurement (442 pc) erroneous and the Gaia DR1 $(176 \mathrm{pc})$ less likely than that from HipPARCOS (133 pc) since this estimate also matches the average $(129 \mathrm{pc})$ for the other co-moving members of the parent cloud of RY Tau. An investigation of the miscalculation from Gaia is beyond the scope of this paper. We nonetheless note that this may have a connection with the variability of the HIPPARCOS astrometric solution (Bertout et al. 1999; Agra-Amboage et al. 2009) indicating motion of the photocenter, or with the presence of a bright envelope, as in $\mathrm{R}$ CrA (Mesa et al. 2019).

\subsection{Stellar temperature, mass, and age}

Two different estimates of the effective temperature $T_{\text {eff }}$ are commonly adopted in the literature: $T_{\text {eff }}=5080 \mathrm{~K}$ (Kenyon \& Hartmann 1995) and $T_{\text {eff }}=5945 \mathrm{~K}$ (Calvet et al. 2004). This discrepancy motivated a new analysis of the stellar temperature. We obtained an independent estimate of $T_{\text {eff }}$ by comparing the optical spectrum of RY Tau obtained with WHT/UES 

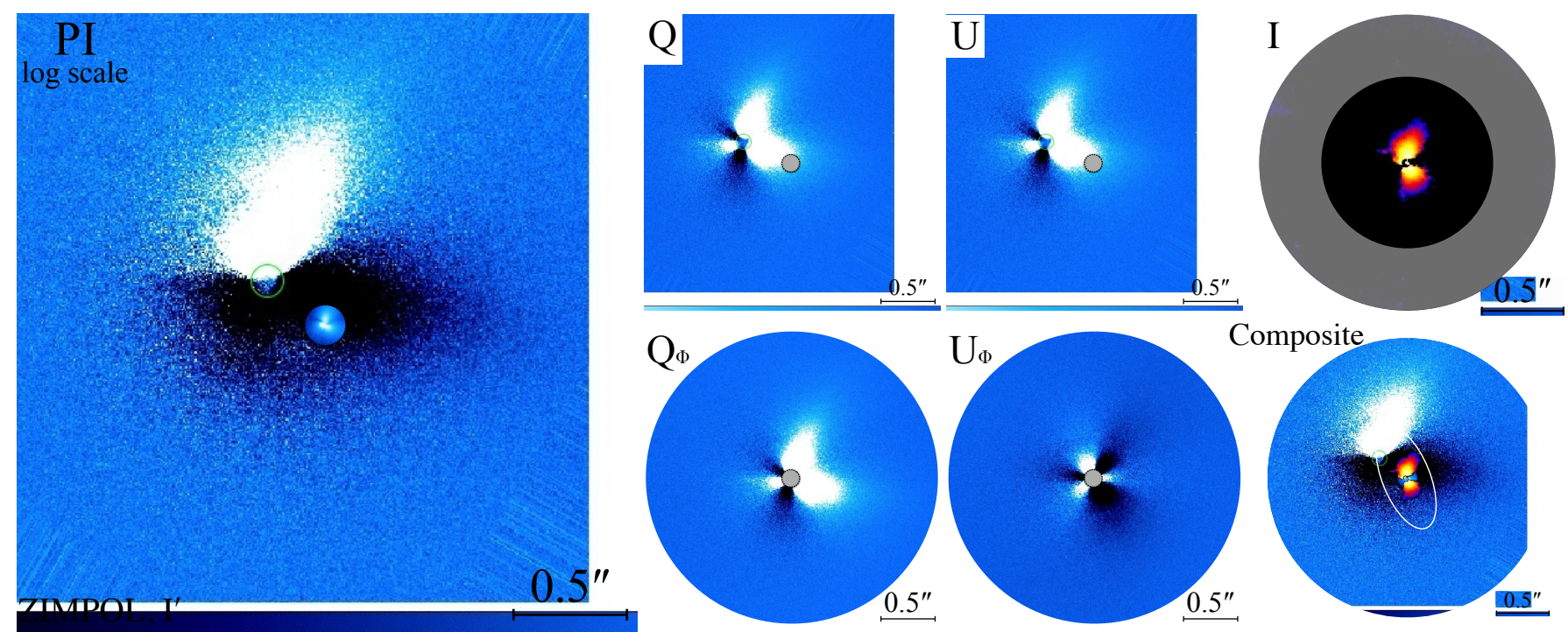

Fig. 1. Imagery of RY Tau in the $I^{\prime}$ band from ZIMPOL. The polarized intensity PI is shown to the left in logarithmic scale with the main region obtained with coronagraphic SlowPol exposures and the small, inner region with FastPol exposures (see text). The $Q, U, Q_{\phi}$, and $U_{\phi}$ parameters are shown in the two central columns with the same linear scale (note the different scale from PI) where black regions indicate negative values. The total intensity image obtained in RDI is shown in logarithmic scale to the top right (the black pixels indicate negative values and the gray mask unreliable results), along with a graphic comparison with PI to the bottom right. The white ellipse outlines the protoplanetary disk seen by ALMA (Long et al. 2018). North is up, east is left.

(see Sect. 2.2) to a set of synthetic models of the stellar photosphere computed from the Atlas and Synthe codes (Kurucz 1993; Sbordone et al. 2004). We varied $T_{\text {eff }}$ and the surface gravity $\log (g)$ and found the best fit from the model with $T_{\text {eff }}=5750 \mathrm{~K}$ and $\log (g)=3.58$ (see Appendix B for details).

We re-calculated all stellar properties using the newly derived $T_{\text {eff }}$ and $d=133 \mathrm{pc}$ (see Sect.3.1). We collected the optical-to-millimeter photometry of the source from the literature. RY Tau is known to show a short (weeks) and a long (years) irregular variability in the visible and NIR (see, e.g., Herbst \& Shevchenko 1999), with variations up to 2.3 mag in the $V$ band. In particular, the brightness is anti-correlated with the linear polarization (Herbst et al. 1994; Oudmaijer et al. 2001), suggesting that the short-term variability is due to transient occultations by circumstellar material (e.g., the UX Ori phenomenon). In this scenario, the maximum observed photometry at a given state of the long-term variability is more representative of the stellar photosphere. We set the benchmark photometry to the upper tertile $V=10.0$ mag since this represents the typical high state of the short (weeks) variability during an intermediate state of the long (years) variability. This choice is further motivated by the discussion in Sect. 5.2.1. The correctness of this approach is also supported by the SED, where the adopted optical brightness is in good agreement with the near- and mid-IR photometry, which is less subject to variability (see Appendix B).

Then, we calculated the visual extinction $A_{\mathrm{V}}$ through the extinction law by Cardelli et al. (1989) with $R_{\mathrm{V}}=3.1$ and from the comparison of synthetic and observed $(V-R),(V-I)$, and $(R-I)$ colors. Given the high stellar variability, we performed this calculation across the entire light curve by Herbst \& Shevchenko (1999) and found that the average $A_{\mathrm{V}}$ calculated over a few tens of measurements decreases from 1.7 to 0.6 with increasing visual brightness (see Fig. B.1). Here we adopted $A_{\mathrm{V}}=1.5 \pm 0.7$ based on the photometry corresponding to the aforementioned $V=10.0 \mathrm{mag}$. The uncertainty derives from the standard deviation of the subsample with $V=10.0 \pm 0.4$ mag. We calculated the stellar luminosity $L_{*}$ by integrating the flux of the model scaled to the de-reddened photometry and obtained $L_{*}=6.3_{-3.2}^{+9.1} L_{\odot}{ }^{2}$. This relatively low luminosity is peculiar of a star slightly older than the typically assumed Taurus age (1-2 Myr). In fact, different sets of PMS tracks (Dartmouth, Mist, Parsec, Dotter et al. 2008; Dotter 2016; Bressan et al. 2012) agree in identifying a stellar mass of $1.9 M_{\odot}$ at an age of 4.5 Myr. These estimates should be taken with caution given the large uncertainties highlighted here and in Sect. 3.1.

\section{Results from VLT/SPHERE}

\subsection{Envelope}

The polarimetric images of RY Tau obtained with ZIMPOL are shown in Fig. 1 . The $Q$ and $U$ parameters do not show the quadrilobe pattern typical of centro-symmetric scattering from protoplanetary disks (see, e.g., Quanz et al. 2011). Instead, two regions with diffuse positive and negative values are found to the north and south. This evident deviation from centro-symmetric scattering may in principle discourage the use of the $Q_{\phi}$ and $U_{\phi}$ parameters, which are typically adopted under the assumption of this type of scattering. Nonetheless, we show these parameters in Fig. 1, where it can be seen that (i) $Q_{\phi}$ consists of two bright wedges to the north and to the west, and three fainter regions to the SE, two of which have negative values (on average $\sim$ five times lower in absolute value than the bright wedge) and (ii) the $U_{\phi}$ image appears as a relatively symmetric alternation of positive and negative regions (with the negative regions being on average $\sim 25 \%$ brighter in absolute value). They therefore significantly diverge from the ideal morphology to be expected in the case of centro-symmetric scattering where $(i) Q_{\phi}$ only contains positive values and (ii) $U_{\phi}$ only contains noise.

2 This uncertainty is obtained from the uncertainty on $A_{\mathrm{V}}$ and on the $\sigma(d)$ of the L1495 cloud sample (5.4 pc, see Sect. 3.1). By adopting the Gaia DR1 measurement of $d=176 \mathrm{pc}$, our calculation yielded $L_{*}=11.2 L_{\odot}$. 


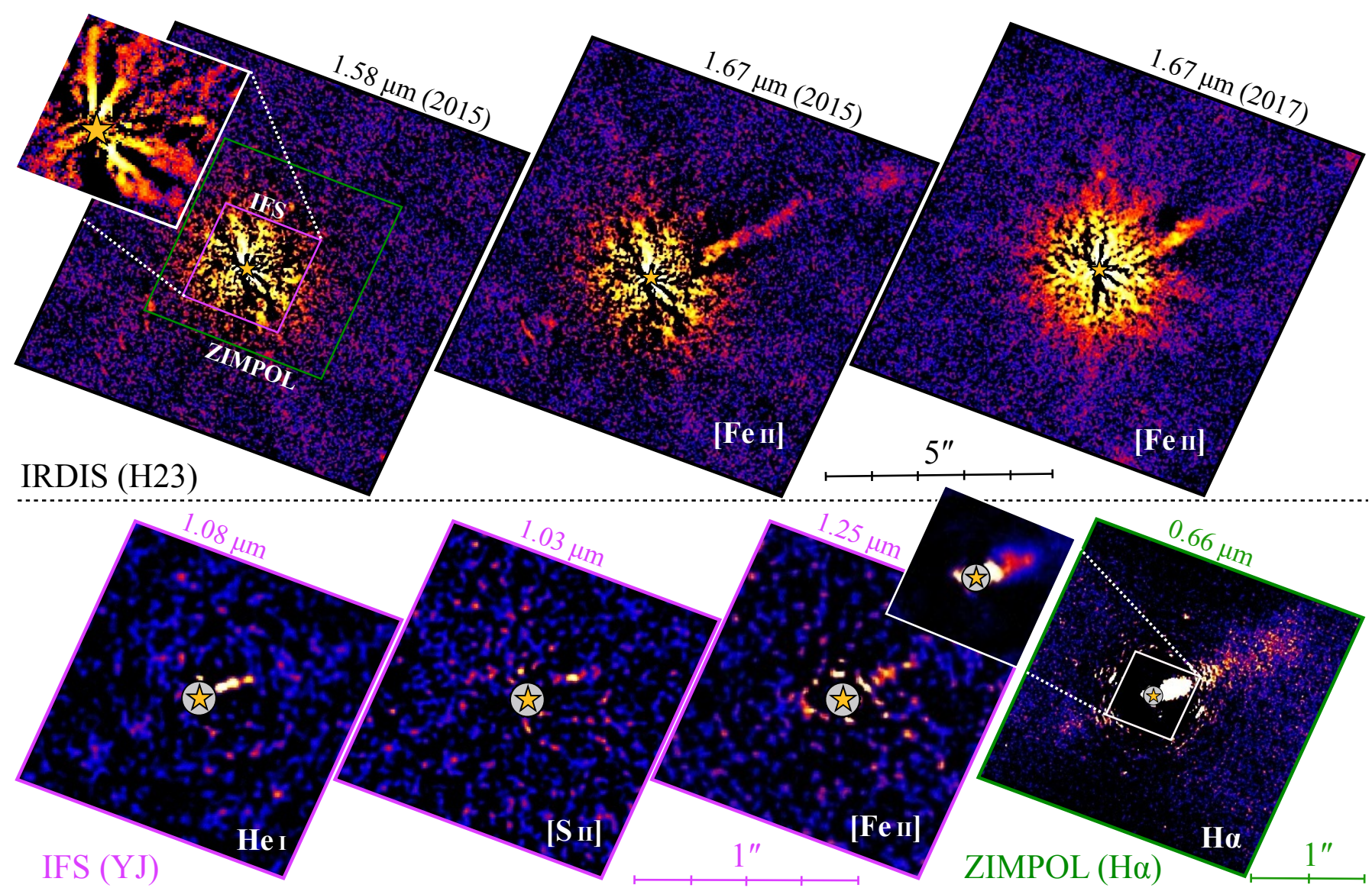

Fig. 2. Imagery of the jet of RY Tau from IRDIS (top), IFS (bottom left), and ZIMPOL (bottom right). The first two IRDIS panels are from 2015, with the left one being in the $H 2$ band and the middle one in the $H 3$ band. Right panel: the H3 image taken in 2017. These images are shown in logarithmic scale with the first two only having the same color stretch. IFS panels are specific channels corresponding to $\lambda=1.08,1.03$, and $1.25 \mu \mathrm{m}$, while the ZIMPOL image is in the $H \alpha$ filter at $0.66 \mu \mathrm{m}$. These images have arbitrary linear color stretch. The inset box of the H $\alpha$ image has a different color stretch and a spatial scale corresponding to the IFS images. The violet box in the top left panel corresponds to the field of the IFS images, while the green box corresponds to the field of the main ZIMPOL image. The stellar position is indicated by the symbol. North is up, east is left.

The combination of the Stokes $Q$ and $U$ parameters into $P I$ (see the left panel of Fig. 1, shown in logarithmic scale) results into a double-wedge structure resembling that seen in the $Q_{\phi}$ image. This morphology is to the first order consistent with the fractional polarization $P I / I$ by Takami et al. (2013), even though a closer inspection reveals that the two wedges from SPHERE are more collimated and their azimuthal distance is smaller. From the inner region of the image (obtained from the FastPolarimetry exposures described in Sect.2), we found no sign of radial discontinuity for the two wedges at least down to the angular resolution of these observations $\left(\sim 0.03^{\prime \prime}\right)$. More importantly, we found nowhere signs of any circumstellar disk features (like gaps, arms, or rings) within the diffuse signal as is recurrently seen in optical and NIR imaging polarimetry of this type of stars. Finally, the disk emission from ALMA by Long et al. (2018) does not have any counterpart in our image (see the bottom right panel of Fig. 1). These considerations and the strong deviation from centro-symmetric scattering suggest that this diffuse scattered light is a residual envelope rather than a disk, in agreement with what was concluded by Takami et al. (2013) (see Sect. 5.1)

The total intensity image obtained in RDI as described in Sect. 2.1 is also shown in Fig. 1 (right column). The only signal that we could retrieve from this procedure is visible as bright double lobes in proximity to the star. It is clear that these lobes match the innermost regions of the extended wedges visible in polarized light. The poor quality of the RDI image with respect to the DPI images discourages the calculation of the polarizedto-total light ratio. It can nonetheless be presumed that the DPI images trace to a large extent the total scattered light distribution and that the observed double-wedge structure is not peculiar of the polarizing properties.

\subsection{Jet}

\subsubsection{Detection of spectral lines}

We investigated four sets of images of RY Tau taken with IRDIS in DBI, two taken in the $H 23$ band and two in the $K 12$ band (see Sect.2). All images show multiple arms that are spatially consistent with the diffuse polarized emission from ZIMPOL (see Sect.4.1). This structure can be seen from Fig. 2 and, in particular, in the inset image of the top left panel, which roughly corresponds to the spatial field of Fig. 2. In addition to this structure, the two available H3 images clearly show evidence of the jet (see the top panels of Fig. 2). This is visible to the NW discontinuously from approximately $1^{\prime \prime}$ out to the detector edge at $6^{\prime \prime}$. There is no sign of the jet from the $\mathrm{H} 2$ images nor from any of the $K 12$ images, suggesting that this emission is the [Fe II] line at $1.64 \mu \mathrm{m}$, which is in fact sitting within the $H 3$ band wavelength coverage (1.61-1.72 $\mu \mathrm{m})$. The $\mathrm{K} 1$ wavelength 
interval includes the $\mathrm{H}_{2}$ line at $2.12 \mu \mathrm{m}$ but this line is not detected. The morphology of both the inner structure and the jet looks very similar from the different post-processing techniques (see Sect. 2 and Appendix A). Thus, in this paper we only focus on the IRDIS images processed with cADI. We nonetheless caution that the process of ADI is known to affect the actual morphology of azimuthally extended structures (see Sect. 4.2.2 for details).

The jet is also detected in 5 of the 39 IFS channels in the $Y J$ band (see the bottom panels of Fig. 2). These channels correspond to $\lambda=1.08 \mu \mathrm{m}$ and $1.03 \mu \mathrm{m}$ and to three consecutive channels around $\lambda=1.25 \mu \mathrm{m}$. Three lines are recurrently detected from jets around these wavelengths: $\mathrm{He}$ I, [S II], and [Fe II], respectively. The same lines are detected in the $Y H$ band but with a lower intensity because the wavewidth of each channel is larger. Thus, we only focus on the $Y J$ dataset. The emission in $\mathrm{He} \mathrm{I}$ is relatively strong and appears as a continuous, narrow feature from the coronagraph edge to $\sim 0.3^{\prime \prime}$, and putatively further out. The [S II] line is barely detected as a bright knot coincident with the western end of the He I main emission. The [Fe II] emission appears faint and more radially diffuse than the other lines. Stacking all other channels yielded no further detectable signal indicating that no other lines of comparable brightness are present in our dataset. All lines are best detected in PCA ADI+SDI (see Sect. 2) in the 2017 dataset and in this paper we only focus on these sets of images.

Finally, we recovered the jet emission from the ZIMPOL/SDI image in the $\mathrm{H} \alpha$ filter at $0.66 \mu \mathrm{m}$ (see Sect. 2). In the inner region, this type of emission appears as a strong narrow feature extending out to $\sim 0.3^{\prime \prime}$ (see bottom right panel of Fig. 2) thus resembling the $\mathrm{He}$ I main emission. Further out, the signal is much fainter and more azimuthally extended (see Sect. 4.2.3). From this image, we recovered a faint emission from the counterjet at $180^{\circ}$ from the main jet.

\subsubsection{Jet proper motion}

From the IRDIS images, the jet appears discontinuous and marginally curved along its radial extent, as can be seen from Fig. 3. Looking at the 2015 dataset, the brightest regions is the inner one (out to $2^{\prime \prime}$, referred to as inner spot), almost no emission is detected from $3.5^{\prime \prime}$ to $5^{\prime \prime}$, but an additional, outer spot is evident from $5^{\prime \prime}$ to $6^{\prime \prime}$. The jet looks rather collimated in the inner region (width $\sim 0.3^{\prime \prime}$ ) while it appears more azimuthally extended (up to $\sim 0.6^{\prime \prime}$ ) further out (see Sect. 4.2.3). A small bend toward the north (left bend) is visible at 3.5" . Similarly, the outer spot appears slightly displaced from the jet axis (assumed at $293^{\circ}$ from the disk geometry, Pinilla et al. 2018). We caution that in principle the process of ADI may bias the morphology of any extended emissions (see, e.g., Garufi et al. 2016; Pohl et al. 2017). This is particularly true for the azimuthal direction since the net effect of the ADI on an azimuthal extended feature is the selfsubtraction. However, this caveat might not have a particularly big impact since the RDI image performed on this dataset looks very similar to the ADI images (see Appendix A). Furthermore, the very good angular resolution of these observations $\left(\sim 0.06^{\prime \prime}\right)$ is such that all these features are well resolved.

The 2017 dataset shows the same inner spot and, marginally, the left bend. Both structures seem to be offset from the 2015 image (see Fig. 3). To quantify this offset, we measured the radial location of the inner and outer edge of the inner spot as well as the jet location, which is most displaced by the jet axis in the proximity of the left bend. This turned out to be $0.31^{\prime \prime} \pm$ $0.03^{\prime \prime}, 0.36^{\prime \prime} \pm 0.03^{\prime \prime}$, and $0.37^{\prime \prime} \pm 0.03^{\prime \prime}$, respectively (with
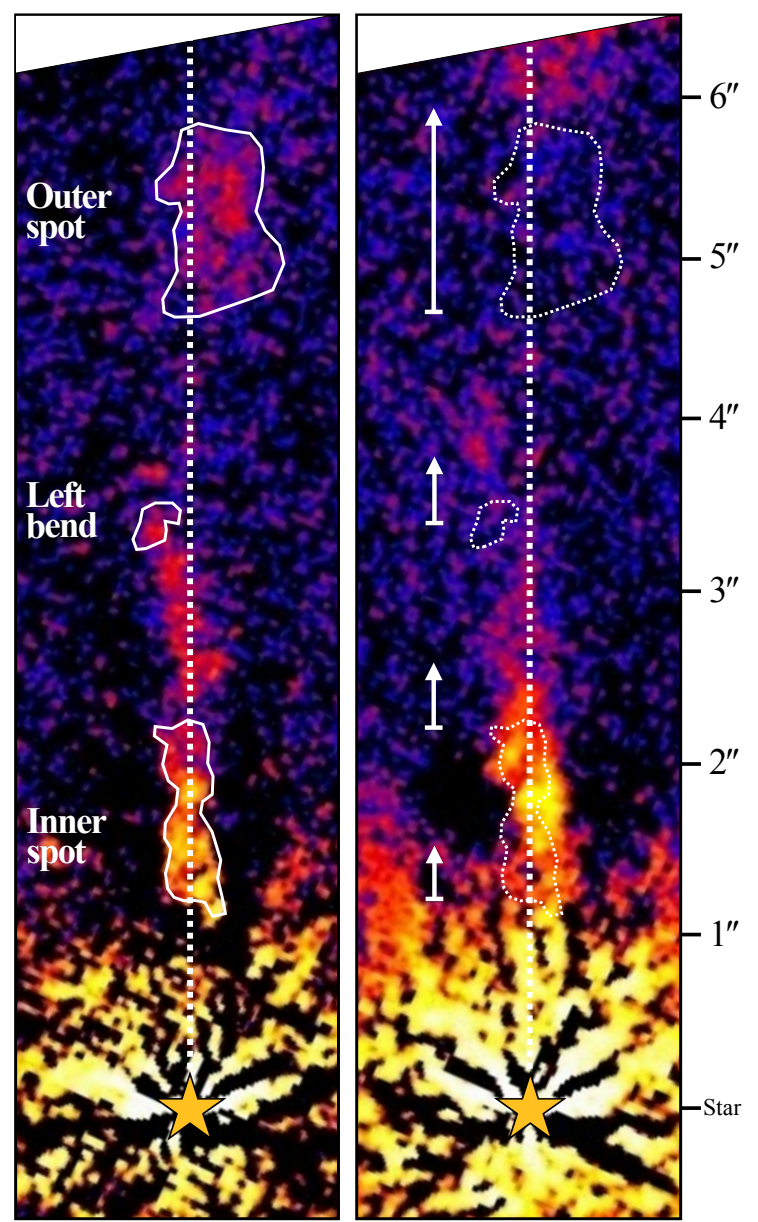

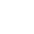




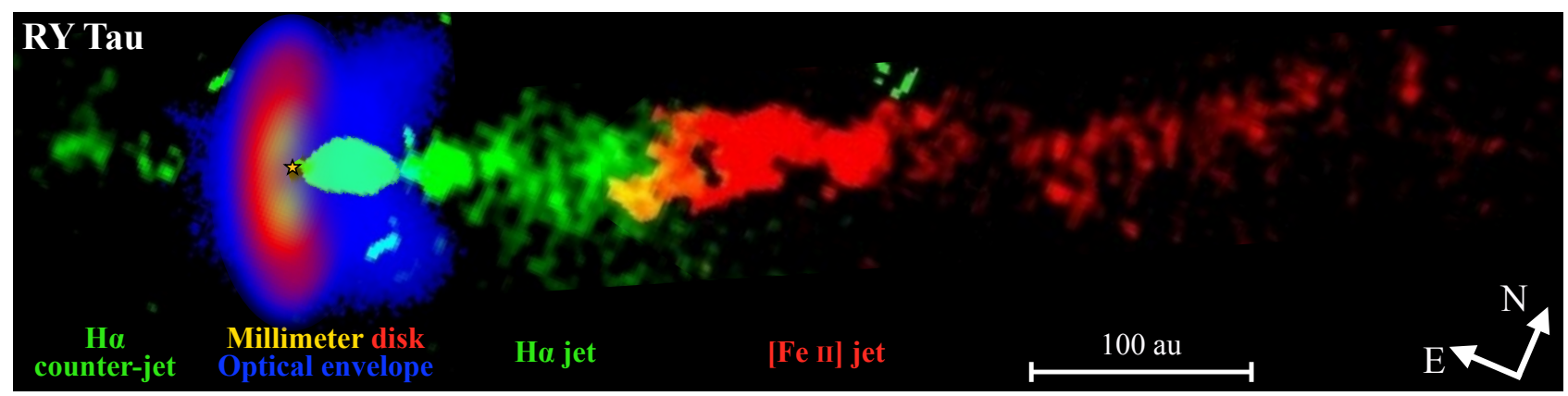

Fig. 4. Composite image of RY Tau comparing the datasets of this work superimposed onto the disk seen in the millimeter by ALMA (Long et al. 2018). Our data consists of the optical envelope of Fig. 1 and of the prominent jet traced by $\mathrm{H} \alpha$ and [Fe II] lines of Fig. 2.

uncertainties adopted as half of the image resolution). It is thus possible that this offset reflects the jet proper motion. Assuming $d=133 \mathrm{pc}$ (see Sect.3.1), these values correspond to a tangential velocity $V_{\mathrm{T}} \sim 90,105$, and $110 \mathrm{~km} \mathrm{~s}^{-1}$, which is a reasonable value for a jet but considerably lower than that inferred by St-Onge \& Bastien (2008) from Gemini and Hubble observations further out $\left(165 \mathrm{~km} \mathrm{~s}^{-1}\right.$ at $\left.6^{\prime \prime}\right)$. The jet radial velocity $V_{\mathrm{R}}$ from [O I] emission is 70-75 $\mathrm{km} \mathrm{s}^{-1}$ (Cabrit et al. 1990; Coffey et al. 2015), while it is much larger inside $1^{\prime \prime}\left(110-150 \mathrm{~km} \mathrm{~s}^{-1}\right.$, Skinner et al. 2018). The jet inclination deriving from our $V_{\mathrm{T}}$ and $V_{\mathrm{R}}=70 \mathrm{~km} \mathrm{~s}^{-1}$ is $i_{\text {jet }}=35^{\circ}$, which is slightly larger than that expected $\left(28^{\circ}\right)$ from the disk geometry (see Pinilla et al. 2018). Interestingly, the 2017 image does not show the outer spot at the location expected from the aforementioned $V_{\mathrm{T}}$. Instead, signal with a similar morphology is revealed at the detector edge. Although only the tail of this feature is visible, it is clear from a close inspection of different epochs and post-processing techniques (see Appendix A) that it corresponds to the outer spot. If so, the $V_{\mathrm{T}}$ of this jet feature is clearly higher than that of the others and we constrained it as $V_{\mathrm{T}} \sim 300 \mathrm{~km} \mathrm{~s}^{-1}$.

Having estimated $V_{\mathrm{T}}$, we calculated the launch date of the inner and outer spots. Assuming that the $V_{\mathrm{T}}$ inferred for the inner spot has been constant with time, this feature turned out to be launched between early 2004 and late 2007. To check for the possible stellar activity level during this period, we retrieved the light curve of RY Tau in the visual waveband over the last $50 \mathrm{yr}$ from the American Association of Variable Star Observers $(\mathrm{AAVSO})^{3}$. Interestingly, the possible launch interval of the inner spot includes the time of the highest photometric state over this period, between 2005 December and 2006 March (see bottom panel of Fig. 3). On the other hand, the launch date of the outer spot is more controversial since we cannot conclude whether its very high $V_{\mathrm{T}}\left(\sim 300 \mathrm{~km} \mathrm{~s}^{-1}\right)$ has been constant or whether it is due to a recent, and possibly temporary, acceleration (see Sect. 5.2.3). Assuming that this feature has traveled at the same velocity of the inner spot $\left(\sim 100 \mathrm{~km} \mathrm{~s}^{-1}\right)$ for most of its lifetime, its launch date corresponds to the other main photospheric maximum registered over the last half a century, in the 1980s (see Fig. 3). Instead, with the currently measured $V_{\mathrm{T}}$ the feature would have a launch date approximately corresponding to that of the inner spot.

In line with such reasoning, we could argue that the faint [Fe II] emission at $1.25 \mu \mathrm{m}$ revealed by IFS in the inner $1^{\prime \prime}$ is due to the very low stellar state registered over the last five years (with visual magnitude spanning from 10.5 to 11, see Fig. 3 for reference). It is thus possible that the $1^{\prime \prime}-5^{\prime \prime}$ region of the jet will look relatively faint over the next decade. The bright emission

WWW . aavso.org revealed in $\mathrm{H} \alpha$ and $\mathrm{He}$ I in the inner region is not necessarily in contrast with this scenario since these lines may be connected to strong stationary shocks in the proximity of the star and, as such, not trace the actual volumes of expanding gas.

\subsubsection{Jet width and wiggle}

The $\mathrm{H} \alpha$ and the He I emission from ZIMPOL and IFS appears bright and compact in the inner $40 \mathrm{au}$. In this region, the jet width measured from these two lines is approximately 12 au and $8 \mathrm{au}$, respectively. The former estimate is more reliable since the ZIMPOL images have a higher angular resolution ( $\sim 4$ au versus $\sim 6 \mathrm{au}$ ) and were not post-processed with ADI. While we do not recover significant $\mathrm{He}$ I emission at larger radii, the $\mathrm{H} \alpha$ emission is detected up to 100-150au where the jet looks much wider, $\sim 40 \mathrm{au}$. The inner spot detected in [Fe II] at 200-250 au has a comparable width, whereas the outer spot at 600 au appears as wide as $\sim 80 \mathrm{au}$. These numbers point to a bottle-neck shape for the jet with an appreciable flow widening occurring between 40 and $100 \mathrm{au}$. Interestingly, these radii correspond to the region of increasing azimuthal divergence between the two DPI wedges (see Fig. 1). This complementarity can also be appreciated from the diagram of Fig. 5 and the composite image of Fig. 4 and it highlights the jet efficiency in sculpting the surrounding medium with its passage.

As commented on in Sect. 4.2.2, the jet orientation changes with the distance from the star. By extracting the center of the azimuthal extension from different tracers and at different radii, the jet position angle (PA) varies from $290^{\circ}$ (from the innermost region and the outer spot) to $295^{\circ}$ (the left bend). Thus, the jet seems to wiggle around the PA expected from the disk orientation (293 ${ }^{\circ}$, Pinilla et al. 2018) and the projected half-opening angle of this wiggle is $2^{\circ}-3^{\circ}$. Since the entire opening angle is found between the left bend and the outer knot (see Fig. 3), we can infer that a wiggle half-cycle elapses over $\sim 300$ au corresponding to $15 \mathrm{yr}$ (with $V_{\mathrm{T}}=100 \mathrm{~km} \mathrm{~s}^{-1}$ ). If the jet wiggle is also present along the radial direction, this effect may partly reconcile the discrepancy between $V_{\mathrm{T}}$ and $V_{\mathrm{R}}$ highlighted in Sect. 4.2.2.

\section{Discussion}

\subsection{Disk and envelope}

Our observations in polarized light described in Sect. 4.1 are indicative of a partly embedded source. In fact, although the stellar photosphere is visible and the SED is characteristic of Class II objects (Lada 1987), the disk revealed in the sub-millimeter at $\mathrm{PA}=23^{\circ}$ (see Fig. 4) remains undetected in scattered light. In the context of optical and NIR imaging polarimetry of 


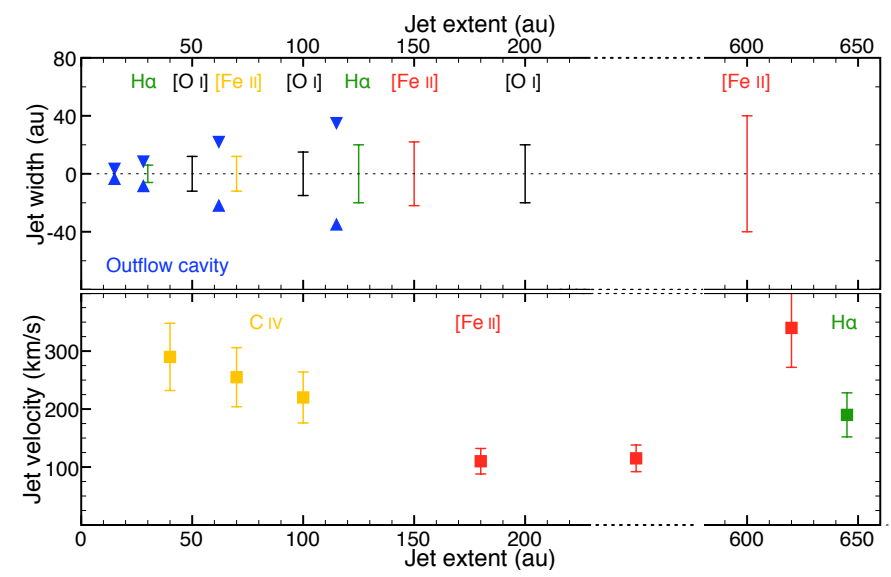

Fig. 5. Jet properties with its radial extent. Top: jet width inferred from different tracers from this work, Agra-Amboage et al. (2009; black), and Coffey et al. (2015; yellow). The blue triangles indicate the outflow cavity size inferred from the PI images in Fig. 1. Bottom: jet velocity inferred from different tracers from this work, Skinner et al. (2018; yellow), and St-Onge \& Bastien (2008; green). The $x$-axis has a discontinuity.

protoplanetary disks, this morphology is uncommon (see Garufi et al. 2018). Similar observations were described by Takami et al. (2013) who reproduced the polarized emission by means of an optically thin and geometrically thick upper layer. This evident nebulosity is likely acting to scatter the NIR photons from the disk but it is not sufficiently thick to mask the stellar photosphere. In other words, it represents the last stage of a protostellar envelope.

The spatial analogy between the wedges in the PI image in Fig. 1 and the jet of Fig. 2 is reminiscent of the outflow cavity of earlier evolutionary stages (see, e.g., Arce \& Sargent 2006). In particular, the opening angle measured from the azimuthal distance of our DPI wedges $\left(\sim 80^{\circ}\right.$, considering the edge as the angle where the flux drops below $3 \sigma$ ) is what is expected from $\gtrsim 1$ Myr-old sources (Seale \& Looney 2008) where most of the envelope has been swept away by the jet and a broader wind outflow. This effect can be seen from Figs. 5 and 4, where it is clear that the jet appears less collimated from 50 au outward, that is, where the opening angle between the optical wedges appreciably increases.

It is intriguing that in this scenario of early evolution, the protoplanetary disk already shows an inner dusty cavity (Isella et al. 2010). According to recent ALMA data (see Fig. 4), this cavity is relatively large $(\sim 18 \mathrm{au})$ but also rather shallow, having only a factor of two depletion in dust (Long et al. 2018). The existence of both a disk cavity and a jet is not necessarily odd provided that gas is still flowing within the cavity. The source is in fact accreting $\left(\sim 2.2 \times 10^{-8} M_{\odot} \mathrm{yr}^{-1}\right.$, Mendigutía et al. 2011). It is tempting to speculate that the cavity has formed recently and that this has not impacted yet on the jet launch mechanisms (see also Sect. 5.3).

\subsection{Jet}

As discussed in Sect. 4.2, we detected the jet in the $\mathrm{H} \alpha$, [S II] $1.03 \mu \mathrm{m}, \mathrm{He}$ I $1.08 \mu \mathrm{m}$, and [Fe II] lines at $1.25 \mu \mathrm{m}$ and $1.64 \mu \mathrm{m}$. Previous work on RY Tau has revealed the emission of the $\mathrm{H} \alpha$ and other optical lines (Cabrit et al. 1990; Hartigan et al. 1995; St-Onge \& Bastien 2008; Agra-Amboage et al. 2009) and of the [Fe II] in the NIR (Coffey et al. 2015), whereas the [S II] and the $\mathrm{He}$ I are detected for the first time in this jet.
Such a variety of detected emission lines corresponds to the presence of gas in widely different conditions of ionization and excitation level. This is consistent with the presence of multiple shocks in the beam, as frequently observed in the initial section of stellar jets (see, e.g., the sub-arcsecond maps from DG Tau or HH 30 star Bacciotti et al. 2000; Hartigan \& Morse 2007). Such shock fronts can produce widely different excitation and ionization conditions because of a combination of effects. In fact, each front can have a different propagation velocity because of a different ejection velocity at the jet base, and subsequent merging with the adjacent fronts.

Furthermore, the bow-like shape of most of these fronts produces variations of the shock velocity (which drives the excitation level), as the latter is a function of the local inclination angle of the front with respect to the jet direction (e.g., Hartigan et al. 1987). Finally, even for a plane-parallel shock, the cooling of the gas behind the front produces strong gradients in the gas density, temperature, and ionization, which give rise to lines of very different excitation in a spatially unresolved layer (see, e.g., Hartigan et al. 1994; Nisini et al. 2005). We note, however, that the $\mathrm{He}$ I at $1.08 \mu \mathrm{m}$ has a high excitation energy $(\sim 20 \mathrm{eV})$, which means that shock velocities larger than $100 \mathrm{~km} \mathrm{~s}^{-1}$ must be present in the inner $0.5^{\prime \prime}$ of the jet if this line is collisionally excited (see tables in Hartigan et al. 1987, and Sect. 5.2.2).

Our analysis on the jet of RY Tau revealed that:

1. The activity stage of RY Tau might have a temporal connection with the launch of the jet spots.

2. The jet is very collimated ( $\sim 12$ au width) on spatial scales of less than 40 au from the star.

3. The inner part $(\lesssim 500 \mathrm{au})$ of the jet is currently moving as a rigid body with $V_{\mathrm{T}} \sim 100 \mathrm{~km} \mathrm{~s}^{-1}$. However, the outer spot at $\sim 650$ au appears to travel three times faster.

4. The jet wiggles over a period of $30 \mathrm{yr}$ with an opening angle of $\sim 5^{\circ}$.

\subsubsection{Episodic accretion and ejection}

Young stars are known to undergo stages of episodic accretion. The physical origin of these episodes is highly debated but it is likely related to the intrinsic inability of the inner disk to accrete at a steady state, to density perturbations within the disk, or to variability of the magnetic field (see the review by Armitage 2015). In magneto-centrifugal models, accretion and ejection activities are predicted to be coupled (see the review by Konigl \& Pudritz 2000), and the jet bears the record of these episodic accretions.

In view of this, the likely temporal analogy between the jet spot launching and the state of RY Tau points toward an increased mass outflow rate that would in turn be connected to an episodic accretion that occurred in 1985 and 2006 (see Fig. 3). Similar behavior was shown in another well-known jet source, HD 163296. Unlike RY Tau, this Herbig star has been photometrically stable over many decades. However, a few photometric dimming events (Ellerbroek et al. 2014) have been ascribed to the episodic obscuration from circumstellar dusty clouds while optical and NIR brightening events (Sitko et al. 2008) have been ascribed to the periodic lifting of dusty material into the disk wind or halo (Vinković et al. 2006; Bans \& Königl 2012).

Both these effects are more erratic in RY Tau (see Petrov et al. 1999) but the physics responsible for this behavior is likely to be the same. The younger age of RY Tau ( $\sim 5$ vs. $10 \mathrm{Myr})$, the apparently earlier stage of the circumstellar material morphology, and the similar mass of the two stars may suggest that 
RY Tau and HD 163296 represent different stages of a similar evolutionary path.

\subsubsection{Jet collimation and recollimation shock}

The unprecedented resolution of these images ( $\sim 30$ mas for ZIMPOL and 40-60 mas for IRDIS/IFS) and the detection of multiple jet tracers allow an accurate measurement of the jet width from 40 to $600 \mathrm{au}$. The jet opening angle derived from the estimates of Sect. 4.2.3 is $\sim 3.5^{\circ}$. This opening angle is consistent with the widening due to a highly supersonic jet propagating with an average velocity of $150 \mathrm{~km} \mathrm{~s}^{-1}$ and with an internal sound speed of about $\sim 10 \mathrm{~km} \mathrm{~s}^{-1}$ (assuming a typical gas temperature of $10^{4} \mathrm{~K}$ ). This indicates that the jet collimation occurs on spatial scales smaller than 50 au as predicted by magnetocentrifugal models (e.g., Pudritz et al. 2007) and observed in a few other jets from TTSs imaged by Hubble Space Telescope (HST) with 0.1"'-resolution (see, e.g., Cabrit 2007). In all cases, including RY Tau, the measured widths are consistent with those imposed by the collimation via magnetic hoop stresses in models of disk winds, as illustrated for example in Fig. 2 of Ray et al. (2007). The magnetically collimated jet excavates the surrounding medium in its passage, as it results from the complementary morphology of the outflow cavity shown in Sect. 4.2.3.

Interestingly, the innermost 50 au from the star where the jet is already collimated show a clear emission in the $\mathrm{He}$ I line at $1.08 \mu \mathrm{m}$. Given its high excitation energy $(\sim 20 \mathrm{eV})$, the He I emission can be produced either collisionally (if the gas is heated up to $2.5 \times 10^{4} \mathrm{~K}$ ) or by recombination (if the gas is highly ionized). In turn, these scenarios require a high velocity shock and/or a source of ionizing radiation. This is consistent with the detection of faint X-ray emission extending a few arcseconds along the jet since this probes hot plasma $\left(T \sim 10^{6} \mathrm{~K}\right.$, Skinner et al. 2011), and of other high excitation lines detected at UV wavelengths close to the driving source, like the $\mathrm{C}$ IV doublet at $1548 / 1550 \AA\left(T \sim 10^{5} \mathrm{~K}\right.$, Skinner et al. 2018), and the C III $1908 \AA$ and S III $1892 \AA$ lines $(T \sim 3 \times$ $10^{4} \mathrm{~K}$, Gómez de Castro \& Verdugo 2001).

The detection of the $\mathrm{He}$ I line at $1.08 \mu \mathrm{m}$ and of other high excitation lines might also be the signature of the socalled recollimation shock that forms when an initially wide wind is collimated into a jet, likely by magnetic fields (see, e.g., Bonito et al. 2011; Frank et al. 2014). Previous detections of the He I line at $1.08 \mu \mathrm{m}$ have been reported around a large sample of TTSs (Edwards et al. 2006) but the emission was unresolved. However, for DG Tau it was shown through a spectro-astrometric analysis that the He I emission was elongated along the PA of the blue-shifted outflow lobe, and it was kinematically associated with the resolved region bright in [Fe II], which traces the collimated jet (Takami et al. 2002). In the case of our AO observations of RY Tau, He I emission is spatially resolved, it is clearly elongated, and is likely spatially coincident with the first $\sim 50$ au of the collimated jet seen in $\mathrm{H} \alpha$ and in the other forbidden lines. This confirms that the energetic shocks giving rise to the He I emission are related to the outflow activity.

\subsubsection{Jet velocity}

The velocity inferred in Sect.4.2.2 (a de-projected $\sim 105-125 \mathrm{~km} \mathrm{~s}^{-1}$ ) is quite moderate for a jet (see Frank et al. 2014). Skinner et al. (2018) noticed that the jet velocity of RY Tau rapidly decreases from $300 \mathrm{~km} \mathrm{~s}^{-1}$ at 40 au to $240 \mathrm{~km} \mathrm{~s}^{-1}$ at $100 \mathrm{au}$. Interpolating their trend yields the velocity that we inferred for the region outside 150 au (see Fig. 5). Furthermore, the inner spot and the left bend of Fig. 3 can be presumably recognized in the GEMINI-Near-Infrared Integral Field Spectrograph (NIFS) image taken by Coffey et al. (2015) from the same tracer in 2009. The observed radial offset results in an intrinsic velocity of $\sim 125 \mathrm{~km} \mathrm{~s}^{-1}$, which is consistent with our measurement. Interestingly, the spatial interval of the rapid jet braking (from 40 to $150 \mathrm{au}$ ) corresponds to the abrupt increase of jet width (see Sect.5.2.2 and Fig.5), suggesting that the jet velocity and width are connected.

The very different velocity of the outer spot $\left(\sim 340 \mathrm{~km} \mathrm{~s}^{-1}\right)$ with respect to the inner jet is controversial. It could be possibly associated to a bright knot at $1.3^{\prime \prime}$ detected in the [O I] by Agra-Amboage et al. (2009) in 2002. Its average velocity from 2002 to 2015 would be $\sim 215 \mathrm{~km} \mathrm{~s}^{-1}$, which is an intermediate value between the estimated velocities of inner and outer jet. In principle, an abrupt decrease of the ambient density acts to accelerate the jet. It can be calculated from Eq. (5) of Raga et al. (1998) that a three-times-faster jet requires an $\sim 15-50$ times less dense medium (in $\sim 150 \mathrm{au}$ ). The jet velocity obtained slightly outside of our outer spot by St-Onge \& Bastien $\left(2008,190 \mathrm{~km} \mathrm{~s}^{-1}\right)$ instead only requires a factor of five-ten of density decrease (in $\gtrsim 300 \mathrm{au}$ ).

\subsubsection{Disk warp}

The jet wiggle inferred in Sect.4.2.3 can have two major origins, namely $(i)$ the presence of a stellar companion massive enough to induce an orbital motion for the primary star (Anglada et al. 2007) and (ii) the precession of an inner disk portion with respect to the outer disk (Zhu 2019). In the former case, the launching area of the jet moves as the primary star orbits the center of mass. The secondary star must therefore be rather massive. We employed Eqs. (7) and (9) of Anglada et al. (2007) to infer the mass of a putative companion necessary to induce the observed jet wiggle. Using our observed period of the wiggle, half-opening angle (see Sect.4.2.3), and jet velocity (see Sect.4.2.2), the calculation yielded an object of mass $M_{\mathrm{c}}=$ $1.1 M_{\odot}$ at a separation of 14 au from RY Tau (corresponding to $\left.\gtrsim 0.1^{\prime \prime}\right)$. However, such an object would be easily recovered from our intensity images. In general, although the presence of a stellar companion has been claimed by several authors (e.g., Bertout et al. 1999; Nguyen et al. 2012) this has not been firmly demonstrated, and RY Tau is still considered as a single star by recent mid-IR interferometric campaigns (e.g., Varga et al. 2018).

All this said, the precession of a disk warp or broken inner disk (e.g., Min et al. 2017; Facchini et al. 2018) seems a more likely explanation for the jet wiggle. This scenario may also require the presence of a companion, although it does not need to be so massive to alter the orbital motion of RY Tau. As an alternative to a companion, a disk warp may also be induced by the disk misalignment with the stellar magnetic axis (see, e.g., Bouvier et al. 1999) but in this scenario the precessing timescale is related to the stellar rotation and is thus too rapid to explain our jet wiggle. Instead, we focused on the companion scenario and constrained the mass $M_{\mathrm{c}}$ and orbital radius $R_{\mathrm{c}}$ of this putative companion. We assumed that the jet wiggle is a direct consequence of a precessing disk warp and employed the analytical Eq. (27) by Zhu (2019), that can be re-written as

$T_{\text {pre }}=\frac{8}{3 q \cos (i)}(1+q)^{1 / 2}\left(\frac{G M_{*}}{R_{\mathrm{c}}^{3}}\right)^{-1 / 2}\left(\frac{R_{\mathrm{c}}}{R_{\mathrm{d}}}\right)^{3 / 2}$,

where $T_{\text {pre }}$ is the precession timescale, $M_{*}$ is the mass of RY Tau, $q$ is $M_{\mathrm{c}} / M_{*}, i$ is the angular momentum vector misalignment between the companion orbit and the disk, and $R_{\mathrm{d}}$ the location of the gap induced by the companion. Based on the properties of 


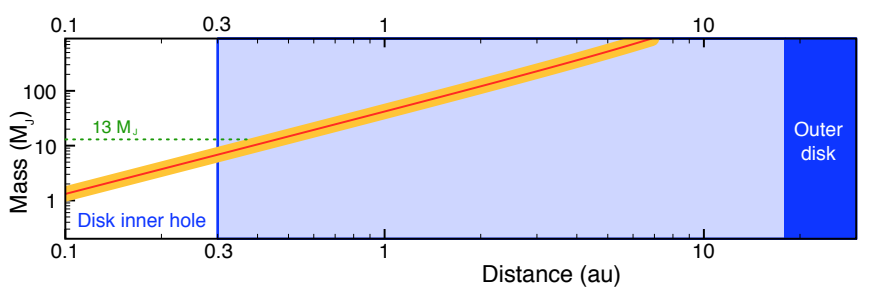

Fig. 6. Mass and orbital distance of a putative companion responsible for the jet wiggle in RY Tau. The yellow region indicates the analytical error bars. The cyan region is between the inner edge of the outer disk from ALMA data (18 au) and the inner edge of the inner disk from midIR interferometric data $(0.3 \mathrm{au})$. The green dashed line is the formal threshold between giant planet and brown dwarf.

the jet wiggle, we imposed $i=5^{\circ}, T_{\text {pre }}=30 \mathrm{yr}$, and assumed that $R_{\mathrm{c}} \gtrsim R_{\mathrm{d}}$. This left us with the family of solution shown in Fig. 6. It turned out that a giant planet inside the disk inner hole (0.3 au, Schegerer et al. 2008) or a substellar companion further out could account for the observed jet wiggle. In the latter case, the inferred companion would be massive enough to tidally break the inner and outer disk (e.g., Pinilla et al. 2012) but may be small enough to remain undetected in high-contrast imaging and interferometric campaigns. This is particularly true in the inner 2-3 au where our calculation yielded $M_{\mathrm{c}}<0.1 M_{\odot}$.

\subsection{Jets of other intermediate-mass stars}

The occurrence of a known jet around Class II intermediate-mass stars is relatively low (see, e.g., Corcoran \& Ray 1997; Eisloffel et al. 2000). In this regard, RY Tau is a fairly exceptional object. In fact, the only other such objects within 200 pc with a direct detection of jet-tracing optical/NIR forbidden lines are, to our knowledge, HD 163296 (Devine et al. 2000), MWC480 (Grady et al. 2010), and HD 104237 (Grady et al. 2004). Although these four stars are fairly similar in mass $\left(\sim 2 M_{\odot}\right)$, their structure is not necessarily identical. Unlike the others, RY Tau is in fact a TTS and as such still possesses a convective envelope that sustains an axisymmetric magnetic field with strong dipole components (Gregory et al. 2012).

In Fig. 7, we compare RY Tau with the sample of nearby intermediate-mass stars with resolved NIR observations of the disk (selected from Garufi et al. 2018, with the addition of HD 104237). In this sample, albeit not complete in any physical sense, only $12 \%$ of the source is known to host a jet. We must nonetheless caution that the detection of a jet in intermediate-mass stars is intrinsically hampered by the bright central continuum emission. However, some considerations on the diagram can be made. First of all, those of HD 163296 and MWC480 are by far the most massive full disks (i.e., hosting no central cavity) in the sample ${ }^{4}$. Formally speaking, the disk of RY Tau is not a full disk (Long et al. 2018) but it clearly differs from the other transition disks of this sample. In fact, the SED does not show evidence of a large cavity and the source is still clearly embedded in the natal cloud (see Sect. 5.1). On the other hand, the possible connection between the disk mass and the presence of a jet is not supported by HD 104237, its disk being in the low tail of dust mass distribution. Figure 7 also shows that there is no clear connection between the presence of the jet and the accretion rate or the stellar mass. In particular, the accretion rate of the four jet sources is in fact relatively high, spanning from $3 \times 10^{-8} M_{\odot} \mathrm{yr}^{-1}$ to $2 \times 10^{-7} M_{\odot} \mathrm{yr}^{-1}$ in a sample

4 Contrary to the literature, HD 142666 is treated as a disk with a cavity, based on Rubinstein et al. (2018).

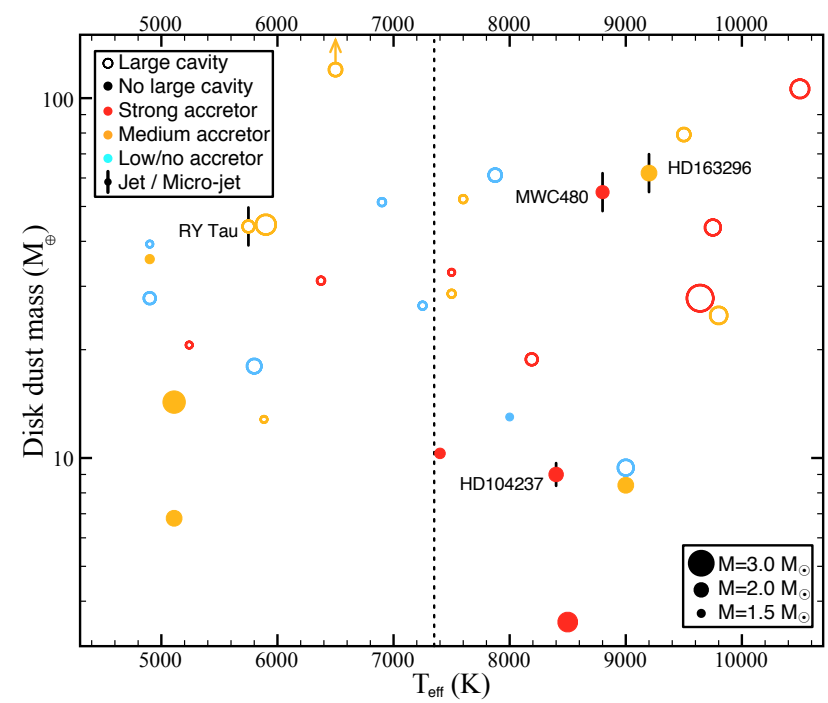

Fig. 7. Disk dust mass vs. effective temperature for a representative sample of intermediate-mass stars (see Garufi et al. 2018). Empty symbols indicate the presence of a disk cavity while the large and small horizontal bars indicate the presence of a known jet and micro-jet, respectively. Symbol sizes are proportional to the stellar mass with dynamic range from 1.5 to $3 M_{\odot}$ while the accretion rate is indicated by the color with strong accretors $\left(>4 \times 10^{-8} M_{\odot} \mathrm{yr}^{-1}\right)$ in red, medium accretors (down to $10^{-8} M_{\odot} \mathrm{yr}^{-1}$ ) in orange, and low/no accretors in cyan. The dashed line separates TTSs and Herbig stars according to the formal threshold at F0 type.

with an average of $4 \times 10^{-8} M_{\odot} \mathrm{yr}^{-1}$ (that does not include 3 out of 32 non-detections). However, other stronger accretors are not known to possess a jet.

RY Tau is the only IMTTS (over 14) showing a jet, while 3 (out of 18) Herbig AeBe are known to host a jet. Bearing in mind the very low statistics, this trend does not support a scenario where jets originate from the stellar surface (Sauty \& Tsinganos 1994), unless the magnetic fields of Herbig stars are much stronger than is currently believed (e.g., Hubrig et al. 2018). On the other hand, a disk-wind scenario where the jet is launched by the magnetic field threading the disk (e.g., Blandford \& Payne 1982; Konigl \& Pudritz 2000) does not require a highly magnetized star. Instead, the presence of a full disk is a condition that intuitively favors this mechanism since the launching region of an atomic jet is the inner 4-5 au (Bacciotti et al. 2002; Coffey et al. 2004). Furthermore, the presence of prominent jets in RY Tau, MWC480, and HD 163296 could indicate that more massive disks are more efficient at launching powerful jets whereas less massive disks (that can be as small as $10 \mathrm{au}$, see Garufi et al. 2017) may only launch a micro-jet (like HD 104237, Grady et al. 2004) or not launch any jet at all.

The absence of other known nearby IMTTS with a jet could also be a selection bias. In fact, Garufi et al. (2018) noticed a desert in the distribution of imaged IMTTS younger than 4-5 Myr and argued that this could be due to the embedded nature of these sources making them a challenging target for AO-driven observations. The intermediate nature of RY Tau discussed in Sect.5.1 is consistent with this scenario. Stars with $M \sim 2-3 M_{\odot}$ may be concealed for several millions of years and then rapidly disperse the envelope and lose the jet thus as to appear like the recurrently imaged intermediate-mass stars older than $~ 5$ Myr.

Finally, RY Tau lies in a rather dense environment, being one of the few intermediate-mass stars in the Taurus cloud. This is in contrast with the majority of the evolved intermediate-mass stars 
observed thus far and could speculatively mean that the interstellar matter accretes onto the protoplanetary disk for a longer timescale than occurs in the isolated Herbig stars. This scenario would reconcile the apparent discrepancy between the early stage of the circumstellar environment and the relatively old age of RY Tau, and thus explain how the jet is sustained for a long timescale.

\section{Summary and conclusions}

The intermediate-mass $\left(M \sim 1.9 M_{\odot}\right)$ star RY Tau has been studied with VLT/SPHERE optical and NIR images. In contrast to the vast majority of the PMS stars available from the literature, our scattered-light images do not reveal the protoplanetary disk that is visible at millimeter wavelengths. Instead, we only resolved the inner region of the remnant envelope, as for previous NIR observations. This nebulosity shows a characteristic dip along the jet axis that is consistent with the late stages of evolution of outflow cavities.

We detected the atomic jet (and marginally the counterjet) with an unprecedented intrinsic spatial resolution (down to $4 \mathrm{au}$ ) in the $\mathrm{H} \alpha$, [S II] at $1.03 \mu \mathrm{m}$, He I at $1.08 \mu \mathrm{m}$, and [Fe II] lines in the 1.25 and $1.64 \mu \mathrm{m}$. Having two observing epochs, we constrained from the proper motion a jet tangential velocity of approximately $100 \mathrm{~km} \mathrm{~s}^{-1}$. From this, we inferred that the launching date for the main jet spot occurred around 2006, when the star experienced a main photometric maximum. The other main stellar brightening of RY Tau occurred in 1985 and can be associated to the launch of an outer spot. This finding is possible evidence of the episodic nature of the accretion process and of the intimate connection between accretion and ejection events.

The jet shows a wiggling, bottle-neck shape with a flow widening of a few degrees between 40 and $100 \mathrm{au}$. The jet morphology in the inner $200 \mathrm{au}$ is consistent with early, strong collimation in a magnetic disk wind, supporting once again the magneto-centrifugal scenario as the origin of jets. We also constrained the mass and orbital distance of a putative companion responsible for the observed jet wiggle. A giant planet at subau scales or a substellar companion further out may in fact induce a disk warp or a misalignment of the inner disk. Such an object may have escaped all planet-search campaigns carried out thus far.

Finally, we put RY Tau in the context of the most studied intermediate-mass stars and noticed that three of the four known jet sources, including RY Tau, host a very massive disk. In particular, HD 163296 and MWC480 host the most massive full disks of the analyzed sample, while the disk of RY Tau only shows a very shallow (possibly recent) cavity that does not leave any imprint on the SED. While it is still possible that the paucity of other IMTTSs with a jet is an observational bias, our small sample of intermediate-mass stars suggests a marginal role for the stellar magnetic field and that more massive disks are more efficient at launching powerful jets. In any case, a deep characterization and a more in-depth statistical analysis of jet-driving sources will be necessary to better constrain the connection between the natal envelope, the protoplanetary disk, and the jet.

This work also serves as a showcase of the capabilities of SPHERE for the search and characterization of optical jets, following previous work on more distant objects (Antoniucci et al. 2016; Schmid et al. 2017). SPHERE allows us to directly image the inner few hundreds au of the jet with a few au resolution, in a framework where only spectral observations or coronagraphic imaging at larger distance have been conducted thus far.
Acknowledgements. We are grateful to Deirdre Coffey, Stefano Facchini, Giuseppe Lodato, Elena Pancino, and Paola Pinilla for the useful discussions, to Feng Long for sharing the ALMA data of RY Tau, and to Benjamin Montesinos for the help with the WHT/UES spectra. We thank the referee, Michihiro Takami, for the insightful comments that improved the manuscript. We also thank the ESO technical operators at the Paranal observatory for their valuable help during the observations. This work has been supported by the project PRIN-INAF 2016 The Cradle of Life - GENESIS-SKA (General Conditions in Early Planetary Systems for the rise of life with SKA). We also acknowledge support from INAF/Frontiera (Fostering high ResolutiON Technology and Innovation for Exoplanets and Research in Astrophysics) through the "Progetti Premiali" funding scheme of the Italian Ministry of Education, University, and Research. F.M. acknowledges funding from ANR of France under contract number ANR-16-CE31-0013. C.P. acknowledges financial support from the ICM (Iniciativa Científica Milenio) via the Núcleo Milenio de Formación Planetaria grant, from the Universidad de Valparaíso. A.Z. acknowledges support from the CONICYT + PAI/ Convocatoria nacional subvención a la instalación en la academia, convocatoria 2017 + Folio PAI77170087. SPHERE is an instrument designed and built by a consortium consisting of IPAG (Grenoble, France), MPIA (Heidelberg, Germany), LAM (Marseille, France), LESIA (Paris, France), Laboratoire Lagrange (Nice, France), INAF - Osservatorio di Padova (Italy), Observatoire de Geneve (Switzerland), ETH Zurich (Switzerland), NOVA (Netherlands), ONERA (France), and ASTRON (Netherlands) in collaboration with ESO. SPHERE was funded by ESO, with additional contributions from the CNRS (France), MPIA (Germany), INAF (Italy), FINES (Switzerland), and NOVA (Netherlands). SPHERE also received funding from the European Commission Sixth and Seventh Framework Programs as part of the Optical Infrared Coordination Network for Astronomy (OPTICON) under grant number RII3-Ct-2004-001566 for FP6 (2004-2008), grant number 226604 for FP7 (2009-2012), and grant number 312430 for FP7 (2013-2016). This work has made use of the SPHERE Data Centre, jointly operated by OSUG/IPAG (Grenoble), PYTHEAS/LAM/CeSAM (Marseille), OCA/Lagrange (Nice), and Observatoire de Paris/LESIA (Paris) and supported by a grant from Labex OSUG@2020 (Investissements d'avenir - ANR10 LABX56). It has also made use of the SIMBAD database, operated at the CDS, Strasbourg, France.

\section{References}

Agra-Amboage, V., Dougados, C., Cabrit, S., Garcia, P. J. V., \& Ferruit, P. 2009, A\&A, 493, 1029

Amara, A., Quanz, S. P., \& Akeret, J. 2015, Astron. Comput., 10, 107

Anglada, G., López, R., Estalella, R., et al. 2007, AJ, 133, 2799

Antoniucci, S., Podio, L., Nisini, B., et al. 2016, A\&A, 593, L13

Arce, H. G., \& Sargent, A. I. 2006, ApJ, 646, 1070

Armitage, P. J. 2015, ArXiv e-prints [arXiv:1509.06382]

Avenhaus, H., Quanz, S. P., Meyer, M. R., et al. 2014, ApJ, 790, 56

Bacciotti, F., Mundt, R., Ray, T. P., et al. 2000, ApJ, 537, L49

Bacciotti, F., Ray, T. P., Mundt, R., Eislöffel, J., \& Solf, J. 2002, ApJ, 576, 222

Bans, A., \& Königl, A. 2012, ApJ, 758, 100

Bertout, C., Robichon, N., \& Arenou, F. 1999, A\&A, 352, 574

Beuzit, J. L., Vigan, A., Mouillet, D., et al. 2019, A\&A, submitted [arXiv:1902.04080]

Blandford, R. D., \& Payne, D. G. 1982, MNRAS, 199, 883

Bonito, R., Orlando, S., Miceli, M., et al. 2011, ApJ, 737, 54

Bouvier, J., Chelli, A., Allain, S., et al. 1999, A\&A, 349, 619

Bressan, A., Marigo, P., Girardi, L., et al. 2012, MNRAS, 427, 127

Cabrit, S. 2007, IAU Symp., 243, 203

Cabrit, S., Edwards, S., Strom, S. E., \& Strom, K. M. 1990, ApJ, 354, 687

Calvet, N., Muzerolle, J., Briceño, C., et al. 2004, AJ, 128, 1294

Carbillet, M., Bendjoya, P., Abe, L., et al. 2011, Exp. Astron., 30, 39

Cardelli, J. A., Clayton, G. C., \& Mathis, J. S. 1989, ApJ, 345, 245

Chauvin, G., Desidera, S., Lagrange, A.-M., et al. 2017, in SF2A-2017: Proceedings of the Annual meeting of the French Society of Astronomy and Astrophysics, eds. C. Reylé, P. Di Matteo, F. Herpin, et al., 331

Claudi, R. U., Turatto, M., Gratton, R. G., et al. 2008, SPIE Conf. Ser., 7014, 3

Coffey, D., Bacciotti, F., Woitas, J., Ray, T. P., \& Eislöffel, J. 2004, Ap\&SS, 292, 553

Coffey, D., Dougados, C., Cabrit, S., Pety, J., \& Bacciotti, F. 2015, ApJ, 804, 2

Corcoran, M., \& Ray, T. P. 1997, A\&A, 321, 189

Delorme, P., Meunier, N., Albert, D., et al. 2017, in SF2A-2017: Proceedings of the Annual meeting of the French Society of Astronomy and Astrophysics, eds. C. Reylé, P. Di Matteo, F. Herpin, et al., 347

Devine, D., Grady, C. A., Kimble, R. A., et al. 2000, ApJ, 542, L115

Dohlen, K., Langlois, M., Saisse, M., et al. 2008, SPIE Conf. Ser., 7014, 3

Dotter, A. 2016, ApJS, 222, 8 
Dotter, A., Chaboyer, B., Jevremović, D., et al. 2008, ApJS, 178, 89 Edwards, S., Fischer, W., Hillenbrand, L., \& Kwan, J. 2006, ApJ, 646, 319

Eiroa, C., Mora, A., Palacios, J., et al. 2000, in Disks, Planetesimals, and Planets, eds. G. Garzón, C. Eiroa, D. de Winter, \& T. J. Mahoney, ASP Conf. Ser., 219,3

Eisloffel, J., Mundt, R., Ray, T. P., \& Rodriguez, L. F. 2000, in Protostars and Planets IV, eds. V. Mannings, A. P. Boss, \& S. S. Russell (Tucson: University of Arizona Press), 815

Ellerbroek, L. E., Podio, L., Dougados, C., et al. 2014, A\&A, 563, A87

ESA 1997, The HipPARCos and Tycho Catalogues. Astrometric and Photometric Star Catalogues Derived from the ESA HipPARCos Space Astrometry Mission, ESA SP, 1200

Facchini, S., Juhász, A., \& Lodato, G. 2018, MNRAS, 473, 4459

Frank, A., Ray, T. P., Cabrit, S., et al. 2014, Protostars and Planets VI (Tucson: University of Arizona Press), 451

Fusco, T., Petit, C., Rousset, G., et al. 2006, SPIE Conf. Ser., 6272, 0

Gaia Collaboration 2016, VizieR Online Data Catalog: I/337

Gaia Collaboration (Brown, A. G. A., et al.) 2018, A\&A, 616, A1

Galicher, R., Boccaletti, A., Mesa, D., et al. 2018, A\&A, 615, A92

Garufi, A., Quanz, S. P., Schmid, H. M., et al. 2016, A\&A, 588, A8

Garufi, A., Meeus, G., Benisty, M., et al. 2017, A\&A, 603, A21

Garufi, A., Benisty, M., Pinilla, P., et al. 2018, A\&A, 620, A94

Gómez de Castro, A. I., \& Verdugo, E. 2001, ApJ, 548, 976

Grady, C. A., Woodgate, B., Torres, C. A. O., et al. 2004, ApJ, 608, 809

Grady, C. A., Hamaguchi, K., Schneider, G., et al. 2010, ApJ, 719, 1565

Gregory, S. G., Donati, J.-F., Morin, J., et al. 2012, ApJ, 755, 97

Hartigan, P., \& Morse, J. 2007, ApJ, 660, 426

Hartigan, P., Raymond, J., \& Hartmann, L. 1987, ApJ, 316, 323

Hartigan, P., Morse, J. A., \& Raymond, J. 1994, ApJ, 436, 125

Hartigan, P., Edwards, S., \& Ghandour, L. 1995, ApJ, 452, 736

Herbst, W., \& Shevchenko, V. S. 1999, AJ, 118, 1043

Herbst, W., Herbst, D. K., Grossman, E. J., \& Weinstein, D. 1994, AJ, 108 1906

Hubrig, S., Jarvinen, S. P., Schöller, M., et al. 2018, ASP Conf. Ser., 518, 218

Isella, A., Carpenter, J. M., \& Sargent, A. I. 2010, ApJ, 714, 1746

Kenyon, S. J., \& Hartmann, L. 1995, ApJS, 101, 117

Kenyon, S. J., Gomez, M., Marzke, R. O., \& Hartmann, L. 1994, AJ, 108, 251

Konigl, A., \& Pudritz, R. E. 2000, Protostars and Planets IV (Tucson: University of Arizona Press), 759

Kurucz, R. 1993, SYNTHE Spectrum Synthesis Programs and Line Data. Kurucz CD-ROM No. 18 (Cambridge, Mass.: Smithsonian Astrophysical Observatory), 1993, 18

Lada, C. J. 1987, in Star Forming Regions, eds. M. Peimbert \& J. Jugaku, IAU Symp., 115, 1

Lindegren, L., Hernández, J., Bombrun, A., et al. 2018, A\&A, 616, A2

Long, F., Pinilla, P., Herczeg, G. J., et al. 2018, ApJ, 869, 17

Luhman, K. L. 2018, AJ, 156, 271

Maire, A. L., Bonnefoy, M., Ginski, C., et al. 2016, A\&A, 587, A56

Marois, C., Lafrenière, D., Doyon, R., Macintosh, B., \& Nadeau, D. 2006, ApJ, 641, 556

Marois, C., Correia, C., Véran, J.-P., \& Currie, T. 2014, in Exploring the Formation and Evolution of Planetary Systems, eds. M. Booth, B. C. Matthews, \& J. R. Graham, IAU Symp., 299, 48

Mendigutía, I., Calvet, N., Montesinos, B., et al. 2011, A\&A, 535, A99

Mesa, D., Bonnefoy, M., Gratton, R., et al. 2019, A\&A, 624, A4

Min, M., Stolker, T., Dominik, C., \& Benisty, M. 2017, A\&A, 604, L10

Mora, A., Merín, B., Solano, E., et al. 2001, A\&A, 378, 116

Nakajima, T., \& Golimowski, D. A. 1995, AJ, 109, 118

Nguyen, D. C., Brandeker, A., van Kerkwijk, M. H., \& Jayawardhana, R. 2012 ApJ, 745, 119

Nisini, B., Bacciotti, F., Giannini, T., et al. 2005, A\&A, 441, 159

Nisini, B., Santangelo, G., Giannini, T., et al. 2015, ApJ, 801, 121

Oudmaijer, R. D., Palacios, J., Eiroa, C., et al. 2001, A\&A, 379, 564

Pavlov, A., Feldt, M., \& Henning, T. 2008, in Astronomical Data Analysis Software and Systems XVII, eds. R. W. Argyle, P. S. Bunclark, \& J. R. Lewis, ASP Conf. Ser., 394, 581

Petrov, P. P., Zajtseva, G. V., Efimov, Y. S., et al. 1999, A\&A, 341, 553

Pinilla, P., Benisty, M., \& Birnstiel, T. 2012, A\&A, 545, A81

Pinilla, P., Tazzari, M., Pascucci, I., et al. 2018, ApJ, 859, 32

Podio, L., Eislöffel, J., Melnikov, S., Hodapp, K. W., \& Bacciotti, F. 2011, A\&A 527, A13

Pohl, A., Benisty, M., Pinilla, P., et al. 2017, ApJ, 850, 52

Pudritz, R. E., Ouyed, R., Fendt, C., \& Brandenburg, A. 2007, Protostars and Planets V (Tucson: University of Arizona Press), 277

Quanz, S. P., Schmid, H. M., Geissler, K., et al. 2011, ApJ, 738, 23
Raga, A. C., Canto, J., \& Cabrit, S. 1998, A\&A, 332, 714

Ray, T., Dougados, C., Bacciotti, F., Eislöffel, J., \& Chrysostomou, A. 2007, Protostars and Planets V (Tucson: University of Arizona Press), 231

Robitaille, T. P., Whitney, B. A., Indebetouw, R., \& Wood, K. 2007, ApJS, 169, 328

Rubinstein, A. E., Macías, E., Espaillat, C. C., et al. 2018, ApJ, 860, 7

Sauty, C., \& Tsinganos, K. 1994, A\&A, 287, 893

Sbordone, L., Bonifacio, P., Castelli, F., \& Kurucz, R. L. 2004, Mem. Soc. Astron. It. Suppl., 5, 93

Schegerer, A. A., Wolf, S., Ratzka, T., \& Leinert, C. 2008, A\&A, 478, 779

Schmid, H. M., Joos, F., \& Tschan, D. 2006, A\&A, 452, 657

Schmid, H. M., Bazzon, A., Milli, J., et al. 2017, A\&A, 602, A53

Schmid, H. M., Bazzon, A., Roelfsema, R., et al. 2018, A\&A, 619, A9

Seale, J. P., \& Looney, L. W. 2008, ApJ, 675, 427

Sheehan, P. D., \& Eisner, J. A. 2017, ApJ, 840, L12

Sitko, M. L., Carpenter, W. J., Kimes, R. L., et al. 2008, ApJ, 678, 1070

Skinner, S. L., Audard, M., \& Güdel, M. 2011, ApJ, 737, 19

Skinner, S. L., Schneider, P. C., Audard, M., \& Güdel, M. 2018, ApJ, 855, 143

Soummer, R., Pueyo, L., \& Larkin, J. 2012, ApJ, 755, L28

St-Onge, G., \& Bastien, P. 2008, ApJ, 674, 1032

Takami, M., Chrysostomou, A., Bailey, J., et al. 2002, ApJ, 568, L53

Takami, M., Beck, T. L., Pyo, T.-S., McGregor, P., \& Davis, C. 2007, ApJ, 670, L33

Takami, M., Karr, J. L., Hashimoto, J., et al. 2013, ApJ, 772, 145

Varga, J., Ábrahám, P., Chen, L., et al. 2018, A\&A, 617, A83

Vigan, A., Moutou, C., Langlois, M., et al. 2010, MNRAS, 407, 71

Vinković, D., Ivezić, Ž., Jurkić, T., \& Elitzur, M. 2006, ApJ, 636, 348

Zhu, Z. 2019, MNRAS, 483, 4221

${ }^{1}$ INAF, Osservatorio Astrofisico di Arcetri, Largo Enrico Fermi 5, 50125 Firenze, Italy

e-mail: agarufi@arcetri.astro.it

2 INAF - Osservatorio Astronomico di Roma, Via Frascati 33, 00078 Monte Porzio Catone (RM), Italy

3 LESIA, Observatoire de Paris, Université PSL, CNRS, Sorbonne Université, Université Paris Diderot, Sorbonne Paris Cité, 5 place Jules Janssen, 92195 Meudon, France

${ }^{4}$ Université Grenoble Alpes, CNRS, IPAG, 38000 Grenoble, France

${ }^{5}$ INAF - Osservatorio Astronomico di Padova, Vicolo dell'Osservatorio 5, 35122 Padova, Italy

${ }^{6}$ Department of Astronomy, University of Michigan, 1085 S. University Ave, Ann Arbor, MI 48109-1107, USA

7 Institute for Particle Physics and Astrophysics, ETH Zurich, Wolfgang-Pauli-Strasse 27, 8093 Zurich, Switzerland

${ }^{8}$ Department of Physics, University of Oxford, Oxford, UK

${ }^{9}$ Institute for Astronomy, University of Edinburgh, EH9 3HJ, Edinburgh, UK

10 Max Planck Institute for Astronomy, Königstuhl 17, 69117 Heidelberg, Germany

${ }^{11}$ Geneva Observatory, University of Geneva, Ch. des Maillettes 51, 1290 Versoix, Switzerland

12 CRAL, CNRS, Université Lyon 1, 9 avenue Charles André, 69561 Saint Genis Laval Cedex, France

${ }^{13}$ Università degli Studi di Padova, dipartimento di Fisica e Astronomia, vicolo dell'osservatorio 3, 35122 Padova, Italy

${ }^{14}$ STAR Institute, University of Liège, Allée du Six Août 19c, 4000 Liège, Belgium

15 Instituto de Física y Astronomía, Facultad de Ciencias, Universidad de Valparaíso, Av. Gran Bretaña 1111, Valparaíso, Chile

${ }^{16}$ Núcleo Milenio Formación Planetaria - NPF, Universidad de Valparaíso, Av. Gran Bretaña 1111, Valparaíso, Chile

17 Aix Marseille Université, CNRS, LAM - Laboratoire d'Astrophysique de Marseille, UMR 7326, 13388 Marseille, France

18 Núcleo de Astronomía, Facultad de Ingeniería y Ciencias, Universidad Diego Portales, Av. Ejercito 441, Santiago, Chile

${ }^{19}$ Escuela de Ingeniería Industrial, Facultad de Ingeniería y Ciencias, Universidad Diego Portales, Av. Ejercito 441, Santiago, Chile 
Appendix A: Observing setting and post-processing techniques

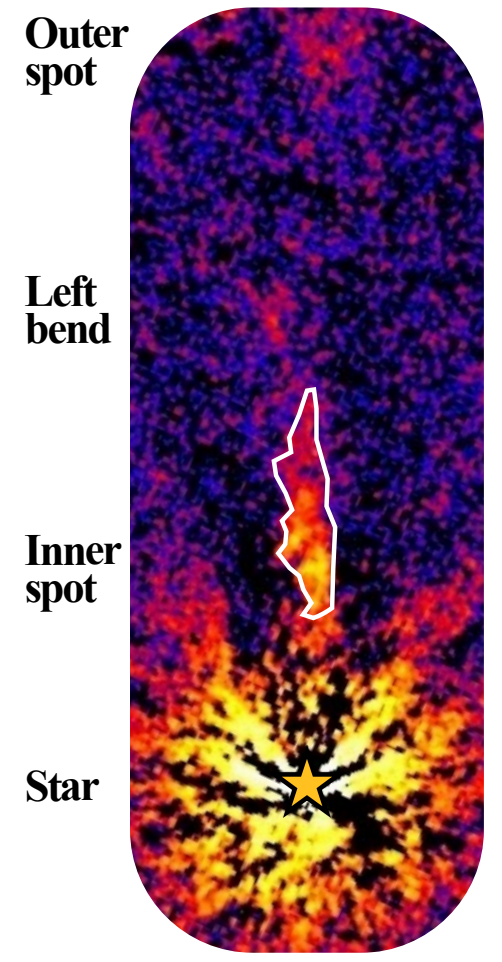

ADI TLOCI

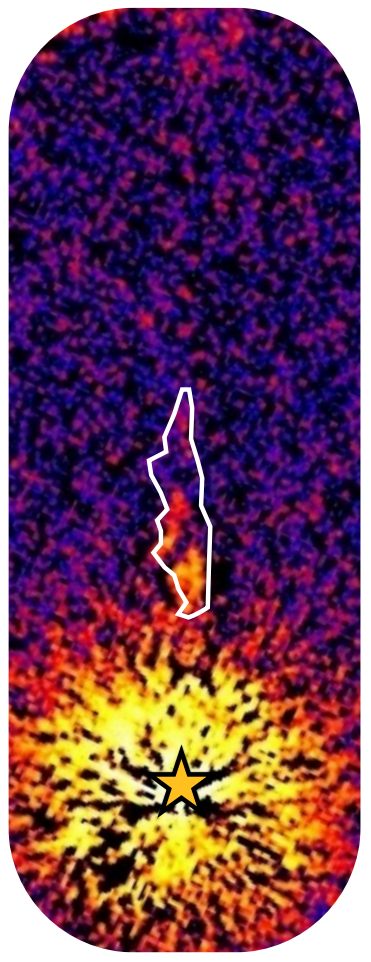

ADI

Fig. A.1. Comparison of different post-processing techniques on the IRDIS H3 images from 2017. The first three figures are obtained in ADI, the fourth in RDI (see Sect. 2). The strong quasi-centrosymmetric signal is the stellar continuum residual. The jet morphology discussed in Sect. 4.2.1 looks reasonably similar (see the white line encircling the inner spot) and all the features that we discussed in the text are always present (with the possible exception of the left bend in the PCA image). Images are rotated by $65^{\circ}$ counterclockwise. The color scale is logarithmic and relatively arbitrary.

Figure A.1 is a comparison of different post-processing techniques on the IRDIS H3 images from 2017. It is clear that all images look reasonably similar, motivating our choice in Sect. 4.2.1 to focus on one image only. In particular, in the RDI image all features have lower contrast than in the ADI images.

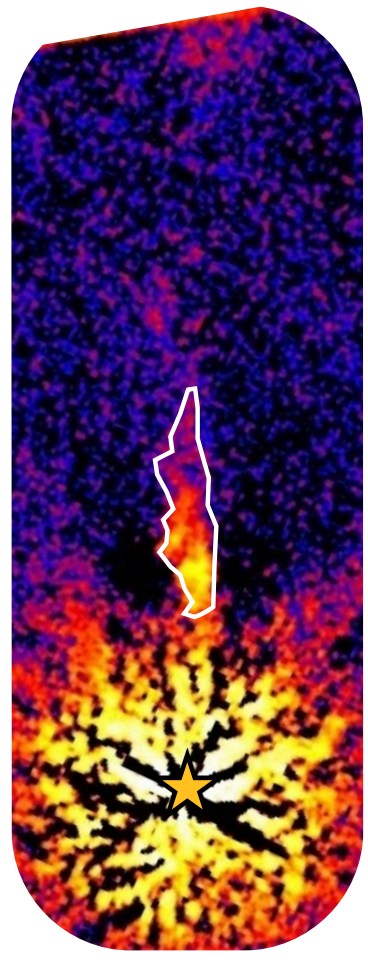

ADI cADI

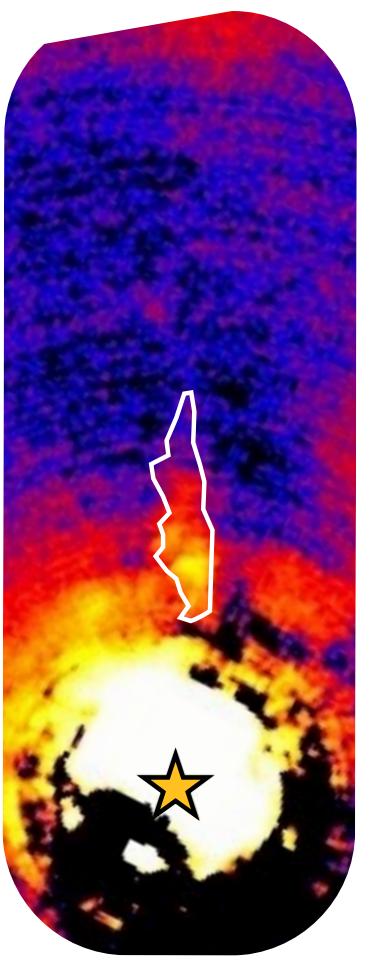

RDI
Their morphology is, however, more reliable since this technique does not suffer from azimuthal self-subtraction as the ADI images do. The similarity with the ADI images suggests that the ADI bias on the jet morphology is limited even in the azimuthal direction. 


\section{Appendix B: Stellar properties of RY Tau}

In Fig. B.1, we show the optical Export spectrum used to constrain the effective temperature of RY Tau (see Sect.3.2), as well as the different values of visual extinction $A_{\mathrm{V}}$, of stellar luminosities, and the SED obtained with different scaling $V$ magnitude (see Sect. 3.2).
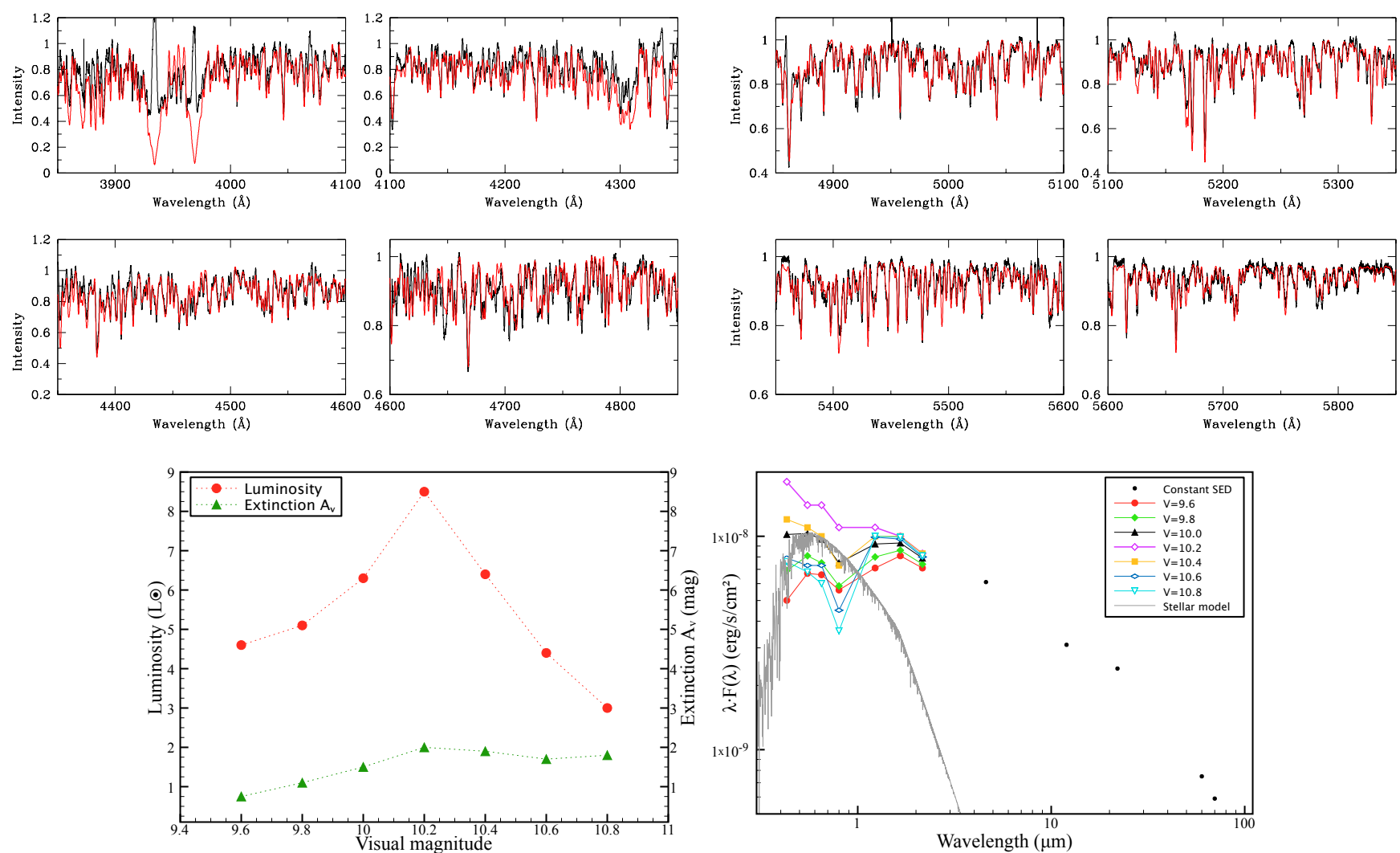

Fig. B.1. Study of the stellar properties of RY Tau. Top panels: the optical EXPORT spectrum (black) compared to a synthetic model (red) with $T_{\text {eff }}=5750 \mathrm{~K}, \log (g)=3.58$, and $v \times \sin (i)=52 \mathrm{~km} \mathrm{~s}^{-1}$. Third panel: the different values of optical extinctions $A_{\mathrm{V}}$ and stellar luminosities obtained by adopting different brightnesses in the $V$ band as a photospheric level. Fourth panel: the de-reddened SED of the source with different values of $V$ mag, and thus $A_{\mathrm{V}}$. A reasonable fit to the optical photometry and agreement with the NIR photometry is obtained only with $V=10.0$ and $V=10.4$. The stellar model is obtained for $V=10.0$ and $A_{\mathrm{V}}=1.5$ and corresponds to a stellar luminosity $L_{*}=6.3 L_{\odot}$. 